\title{
MODELING EXTRAGALACTIC FOREGROUNDS AND SECONDARIES FOR UNBIASED ESTIMATION OF COSMOLOGICAL PARAMETERS FROM PRIMARY COSMIC MICROWAVE BACKGROUND ANISOTROPY
}

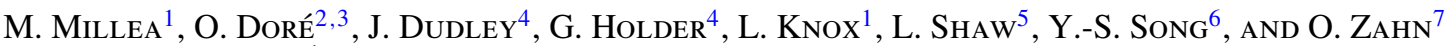 \\ ${ }^{1}$ Department of Physics, University of California, Davis, CA 95616, USA \\ 2 Jet Propulsion Laboratory, California Institute of Technology, Pasadena, CA 91109, USA \\ ${ }^{3}$ California Institute of Technology, Pasadena, CA 91125, USA \\ ${ }^{4}$ Department of Physics, McGill University, Montreal, Quebec H3A 2T8, Canada \\ ${ }^{5}$ Department of Physics, Yale University, New Haven, CT 06520-8120, USA \\ ${ }^{6}$ Korea Institute for Advanced Study, Seoul 130-722, Republic of Korea \\ ${ }^{7}$ Berkeley Center for Cosmological Physics, Department of Physics, University of California, \\ and Lawrence Berkeley National Labs, Berkeley, CA 94720, USA \\ Received 2011 February 25; accepted 2011 October 18; published 2012 January 18
}

\begin{abstract}
Using the latest physical modeling and constrained by the most recent data, we develop a phenomenological parameterized model of the contributions to intensity and polarization maps at millimeter wavelengths from external galaxies and Sunyaev-Zeldovich effects. We find such modeling to be necessary for estimation of cosmological parameters from Planck data. For example, ignoring the clustering of the infrared background would result in a bias in $n_{s}$ of $7 \sigma$ in the context of an eight-parameter cosmological model. We show that the simultaneous marginalization over a full foreground model can eliminate such biases, while increasing the statistical uncertainty in cosmological parameters by less than $20 \%$. The small increases in uncertainty can be significantly reduced with the inclusion of higher-resolution ground-based data. The multi-frequency analysis we employ involves modeling 46 total power spectra and marginalization over 17 foreground parameters. We show that we can also reduce the data to a best estimate of the cosmic microwave background power spectra, with just two principal components (with constrained amplitudes) describing residual foreground contamination.
\end{abstract}

Key words: cosmological parameters - cosmology: observations - cosmology: theory - distance scale large-scale structure of universe

Online-only material: color figures

\section{INTRODUCTION}

The cosmic microwave background (CMB) is arguably the most powerful probe of the parameters and viability of cosmological models. With temperature measurements on angular scales larger than a third of a degree already at the cosmic variance limit (Komatsu et al. 2010), further progress now depends on improvements at smaller angular scales as well as improved measurements of the polarization at all angular scales. Advances in temperature anisotropy measurements come most recently from the Atacama Cosmology Telescope (ACT; Dunkley et al. 2011), soon to be followed by new results from the South Pole Telescope (SPT; Keisler et al. 2011). By the end of 2012 we expect a dramatic improvement from Planck, which is likely to leave very little room for further improvement in the measurement of the primary CMB temperature power spectrum. These improved measurements will translate into much tighter constraints on cosmological models (e.g., Planck Collaboration 2006).

At angular scales smaller than a tenth of a degree, extragalactic foregrounds ${ }^{8}$ become important for three reasons: (1) the CMB power spectrum is dropping in amplitude, (2) cosmic variance is smaller, and (3) foregrounds are growing in amplitude. At sufficiently small angular scales, foregrounds become the dominant signal at all CMB frequencies. Furthermore, unlike galactic foregrounds, they are statistically isotropic and

\footnotetext{
8 We will henceforth refer to both extragalactic foreground contaminants and secondary anisotropies as just "foregrounds" since they cannot be modeled from first principles like the primary $\mathrm{CMB}$.
}

thus cannot be avoided by masking regions of higher contamination. Their modeling is an unavoidable necessity.

In this paper we present a parameterized, physically motivated, phenomenological model for the extragalactic foregrounds and consider it in the context of extracting cosmological parameters from the primary CMB anisotropy. We demonstrate that for an analysis of Planck data, such modeling is necessary to avoid significant biases in cosmological parameter estimates, but that marginalization over even a very rich foreground model is essentially "for free"; the foregrounds are sufficiently orthogonal to the primary CMB that the statistical errors on cosmological parameters are degraded by at most $20 \%$ for $n_{s}$ and less than $10 \%$ for other parameters. With the addition of higher resolution ground-based data or non-CMB Planck bands to clean the foregrounds, the degradation is reduced to a few percent for all parameters.

The importance of extragalactic foregrounds for CMB analysis has been recognized for a long time (Tegmark \& Efstathiou 1996; Bouchet \& Gispert 1999; Knox 1999; Tegmark et al. 2000; Leach et al. 2008; Cardoso et al. 2008; Dunkley et al. 2011). Potential biases from extragalactic contaminants have been pointed out previously by Knox et al. (1998), Santos et al. (2003), Zahn et al. (2005), Serra et al. (2008), and Taburet et al. (2009). Distinguishing our work is the simultaneous consideration of all foreground components necessary for an analysis of Planck data, and physical modeling of these components informed from recent measurements beyond the damping tail by SPT (Hall et al. 2010; Vieira et al. 2010; Shirokoff et al. 2010) and ACT (Dunkley et al. 2011). In this paper, we will consider the foreground power contributions from shot 
noise due to radio galaxies and dusty star-forming galaxies (DSFGs), the clustering of the DSFGs, the thermal and kinetic Sunyaev-Zeldovich effects (tSZ and kSZ), and correlation between the tSZ and DSFG components. We now turn to summarizing recent developments in both modeling and measurements of these extragalactic foregrounds.

Our understanding of the power spectrum due to DSFGs at frequencies relevant for $\mathrm{CMB}$ analysis has been rapidly improving. We demonstrate here that for analysis of Planck data, the effects of DSFG clustering are the most important of the foregrounds to model. Although it is the most important effect, it has been almost entirely ignored by previous cosmological parameter error forecasting work. To date, the only papers to consider the impact of DSFG clustering on cosmological parameter estimates are Dunkley et al. (2011) and Serra et al. (2008).

DSFG clustering power was first detected at CMB frequencies by the SPT (Hall et al. 2010), with subsequent confirmation and improved constraints from ACT (Dunkley et al. 2011) and SPT (Shirokoff et al. 2010). The recent suite of early Planck papers (Planck Collaboration 2011e, in particular) have also provided significant constraints on both the amplitude and shape of the clustering power. The Planck measurements rule out many otherwise viable models which generally predict higher power (on the scales relevant for analysis of the primary CMB power spectrum) than observed.

Radio galaxy source counts from high-resolution groundbased data are particularly useful for Planck since they are sensitive to the decade in brightness below Planck's flux cut. The radio sources in this brightness range create the dominant source of shot noise power in most of the Planck frequencies which contain significant CMB information. SPT measurements of point-source populations (Vieira et al. 2010) have offered valuable information about the amplitude of Poisson power, as well as the coherence of these shot-noise fluctuations from frequency to frequency.

Recent data, as well as recent theoretical developments, inform our modeling of the power spectrum of the tSZ effect-a spectral distortion that arises due to inverse Compton scattering of $\mathrm{CMB}$ photons off the hot electrons in groups and clusters. The magnitude of the tSZ signal is proportional to the thermal pressure of the intracluster medium (ICM) integrated along the line of sight. Upper limits on the amplitude of the tSZ power (set by Lueker et al. 2010, confirmed by Dunkley et al. 2011 and further tightened by Shirokoff et al. 2010) were found to be surprisingly low compared to predictions from halo model calculations (Komatsu \& Seljak 2002) and nonradiative hydrodynamic simulations (White et al. 2002). Recent work has demonstrated that the inclusion of a significant nonthermal contribution to the total gas pressure in groups and clusters in analytic models can significantly reduce the predicted amplitude of the tSZ power spectrum (Shaw et al. 2010; Trac et al. 2010). Non-thermal pressure, sourced by bulk gas motions and turbulence, reduces the thermal pressure required to support the ICM against gravitational collapse and thus the amplitude of the tSZ signal. Similarly, Battaglia et al. (2010) demonstrated that the inclusion of radiative cooling, star formation, and active galactic nucleus (AGN) feedback in hydrodynamic simulations substantially lowers the tSZ power compared to simulations that omit these processes. Current predictions for tSZ power from models and simulations are consistent with the upper limits derived from observations.

These recent modeling developments are supported by data from Planck; when the models are used to extrapolate from
X-ray measurements to a predicted tSZ signal, the predictions agree with Planck SZ observations. Agreement is seen both in observations of single galaxy clusters (Planck Collaboration 2011a, 2011b) and via a stacking analysis over a broad range in X-ray luminosity down to masses as small as $M_{500} \sim$ $5 \times 10^{13} M_{\odot}$ (Planck Collaboration 2011c).

Current data provide no direct lower limits to the amplitude of tSZ power due to a degeneracy with the kinetic SZ power spectrum (Lueker et al. 2010). The kinetic SZ effect arises due to the Doppler Thomson scattering of CMB photons off of regions of ionized gas with bulk peculiar velocities. Upper limits on $\mathrm{kSZ}$ power set by Lueker et al. (2010) and now substantially tightened by Shirokoff et al. (2010), are ruling out some models of patchy reionization. It is useful to decompose the kSZ power into contributions arising from an inhomogeneous transition from a neutral to ionized intergalactic medium, the so-called "patchy reionization," and those from the post-reionization era, the "Ostriker-Vishniac" (OV) effect. The former is much more uncertain than the latter, and our best knowledge of its amplitude comes directly from the upper limits in Shirokoff et al. (2010). The OV power level has a current theoretical uncertainty that we estimate to be about a factor of two. Despite its low levels, $\mathrm{kSZ}$ power is a worrisome source of potential bias of cosmological parameters since its spectral dependence is the same as the primary CMB temperature anisotropies.

We expect that the only potentially significant extragalactic contributions to polarization anisotropy are Poisson power from radio sources and DSFGs. A polarization analog for DSFG clustering could only arise due to (unexpected) correlations between galaxies in the polarization orientations of their emission. Polarization signals arise from scattering off of electrons in clusters and groups (Sazonov \& Sunyaev 1999; Carlstrom et al. 2002; Amblard \& White 2005) and in reionized patches (Knox et al. 1998; Santos et al. 2003), but these are also expected to be negligibly small.

In addition to developing and exploring the implications of an extragalactic foreground model that takes into account recent developments, we introduce a new approach to analyzing the multi-frequency data. We show how the complexities of our modeling can be reduced to a fairly simple description of the contamination of the estimates of CMB power spectra. The contamination can be described by just a few principal components whose amplitudes are constrained by CMB-free linear combinations of the auto and cross-frequency power spectra.

The outline of our paper is as follows. In Section 2 we describe our foreground models before describing our fiducial models and surveys in Sections 3 and 4 respectively. In Section 5 we describe our general methodology before detailing our principal component approach in Section 6. We finally present our results in Section 7 and discuss them in Section 8.

\section{MODELING}

\subsection{Emission from External Galaxies}

In the frequency range in which Planck is most sensitive to the $\mathrm{CMB}$ (roughly $70 \mathrm{GHz}$ to $217 \mathrm{GHz}$ ), external galaxies are well approximated by power-law intensities $I_{v} \propto v^{\alpha}$, and divide fairly cleanly into those with spectral indices $\alpha<1$ (radio galaxies) and those with $\alpha>1$ (DSFGs; Vieira et al. 2010). We assume that all sources have no spatial extent, an approximation which might be worrisome for radio sources because of long relativistic jets. However, if we extrapolate from $1.4 \mathrm{GHz}$ up to 
our frequency range, we find $99 \%$ of sources have a major axis FWHM less than 30 arcsec (Hodge et al. 2011), too small to be detectable with the 1.5 arcmin beams of typical ground-based experiments.

External galaxies lead to anisotropy via their discreteness, usually modeled with a Poisson distribution, and also via correlations due to their tracing of the large-scale structure. The Poisson fluctuations are important for both radio galaxies and DSFGs, while clustering is only significant for the dusty galaxies (Hall et al. 2010).

The Poisson contribution depends on the brightness function, $d N / d S$, via

$$
C_{\ell}=\int_{0}^{S_{c}} d S S^{2} \frac{d N}{d S}
$$

where $S_{c}$ is the flux cut; map pixels with sources with $S>S_{c}$ are masked.

Clustering power, in contrast, scales approximately with the square of the mean intensity, $I_{v}^{2}$, with

$$
I_{v}=\int_{0}^{S_{c}} d S S \frac{d N}{d S} .
$$

Although radio sources do cluster, their mean intensity at the relevant frequencies is much smaller than for the DSFGs; sufficiently smaller that their clustering power is negligible.

\subsubsection{Radio Galaxies}

From Vieira et al. (2010) we know the radio galaxies at $150 \mathrm{GHz}$ and $220 \mathrm{GHz}$ and at flux densities below $100 \mathrm{mJy}$ are described quite well by the de Zotti et al. (2005) model. ${ }^{9}$ This model has a brightness function that is approximately a power-law $S d N / d S \propto S^{\gamma_{R}}$. This translates into Poisson power which depends on the flux cut via $C_{\ell} \propto S_{c}^{\gamma_{R}+2}$. Due to the inhomogeneity of the Planck sky coverage, $S_{c}$ will vary significantly across the sky. So that these angular variations can be taken into account, we chose to model the radio galaxies in terms of $d N / d S$ rather than $C_{\ell}$.

For frequency dependence, we assume the spectral indices of the source population form a Gaussian distribution with mean $\alpha$ and width $\left\langle\delta \alpha^{2}\right\rangle=\sigma^{2}$ (uncorrelated from source to source). With these assumptions our power spectra from radio sources are given by ${ }^{10}$

$$
\begin{aligned}
C_{\ell}^{R}= & C^{R, 0}\left(\frac{S_{c}}{S_{0}}\right)^{\gamma_{R}+2}\left(\frac{\nu \nu^{\prime}}{v_{0}^{2}}\right)^{\left[\alpha_{R}+\ln \left(\nu v^{\prime} / v_{0}^{2}\right) \sigma_{R}^{2} / 2\right]} . \\
& \text { 2.1.2. Dusty Star-forming Galaxies }
\end{aligned}
$$

\subsubsection{Dusty Star-forming Galaxies}

Due the shape of DSFG brightness function, the integrals in Equations (1) and (2) are nearly independent of the upper bound (Hall et al. 2010); thus dusty power is nearly independent of flux cut and we choose to build our model in $C_{\ell}$ rather than $d N / d S$. In that case, the DSFG Poisson contribution is given simply by

$$
C_{\ell}^{D}=C^{D, 0}\left(\frac{v v^{\prime}}{v_{0}^{2}}\right)^{\left[\alpha_{D}+\ln \left(\nu v^{\prime} / v_{0}^{2}\right) \sigma_{D}^{2} / 2\right]}
$$

\footnotetext{
9 We also know from recent Planck results (Planck Collaboration 2011d) that at brighter flux densities the deZotti model significantly overpredicts the number counts.

10 In deriving this form we have used the identity that for a zero-mean Gaussian random variable $x,\langle\exp (-x)\rangle=\exp \left(\left\langle x^{2}\right\rangle / 2\right)$. This identity and its applicability in this context was pointed out to us by Challinor, Gratton, and Migliaccio.
}

A number of authors have considered the clustering of the infrared background, starting with Bond et al. (1986, 1991). Further theoretical investigation (Scott \& White 1999; Haiman \& Knox 2000) was stimulated by the detection of the infrared background in COBE data (Puget et al. 1996; Fixsen et al. 1998), and the detection of bright "submillimeter" galaxies in SCUBA data (Hughes et al. 1998). Subsequently, the clustering has been detected at $160 \mu \mathrm{m}$ (Lagache et al. 2007), at 250, 350, and $500 \mu \mathrm{m}$ by the Balloon-borne Large Aperture Submillimeter Telescope (BLAST; Viero et al. 2009) and at $217 \mathrm{GHz}$ (Hall et al. 2010; Dunkley et al. 2011). Recent Planck measurements of the Cosmic Infrared Background (CIB; Planck Collaboration 2011e) have extended to much larger angular scales than before at $217 \mathrm{GHz}, 353 \mathrm{GHz}, 545 \mathrm{GHz}$, and $857 \mathrm{GHz}$ and recent Herschel measurements (Amblard et al. 2011) have tightened up the BLAST measurements and extended them to smaller angular scales. The field is rapidly evolving.

For the clustering, we assume the same model as in Hall et al. (2010), extended to phenomenologically include the consequences of nonlinear clustering by including a multiplicative factor which is a power law in $\ell$ for $\ell>1500$. This extension is able to fit many different models in the literature and allows us to explore the theoretical uncertainty in a model independent manner. We neglect one aspect of the Hall et al. (2010) model because it leads to corrections of only about $1 \%$ across the relevant frequency range; we ignore the $\ell$-dependent spectral index. Thus, the DSFG clustering power spectra are given by

$$
C_{\ell}^{C}=C^{C, 0} \Phi_{\ell}^{\mathrm{H} 10}\left(\frac{\nu v^{\prime}}{v_{0}^{2}}\right)^{\alpha_{C}} \begin{cases}1 & \ell<1500 \\ \left(\frac{\ell}{1500}\right)^{n_{C}} & \ell>1500\end{cases}
$$

where $\Phi_{\ell}^{\mathrm{H} 10}$ is the Hall et al. (2010) clustering template.

Though the same sources generate both the Poisson power and the clustering power, they are weighted differently; thus for our baseline model we conservatively assume no relationship between the clustering spectral index and the Poisson spectral index.

To gain some idea of the range of possible shapes of the DSFG clustering power spectrum, we show a sampling of power spectra from models in the literature in Figure 1. They are all normalized at $\ell=3000$ to highlight similarities/differences in shape. The models are the fiducial model from Righi et al. (2008), the $\beta=0.6$ model from Amblard \& Cooray (2007) and a nonlinear version of the model by Haiman \& Knox (2000), hereafter HK00. Righi et al. (2008) associate the sources of infrared light with starbursts triggered by mergers. Amblard \& Cooray (2007) incorporate nonlinearities using a halo model. For the "HK00nonlin" curve, we used the luminosity densities for the fiducial model of HK00, assumed that light is a biased tracer of mass, and calculated the nonlinear mass power spectrum using the prescription by Peacock \& Dodds (1996). Though these template arise from very different modeling assumptions, they have similar shapes in the linear regime at large scales, and then turn to a power-law behavior at small scales. It is this observation which informed our phenomenological model. We will also show our model to be sufficient for reproducing these shapes with enough accuracy for Planck cosmological parameter estimation.

One result of the Planck measurements, available only after our calculations for this paper were completed, is that the CIB power spectrum uncertainty at $\ell<2000$ is now much smaller than before. At least two of the three models shown in 


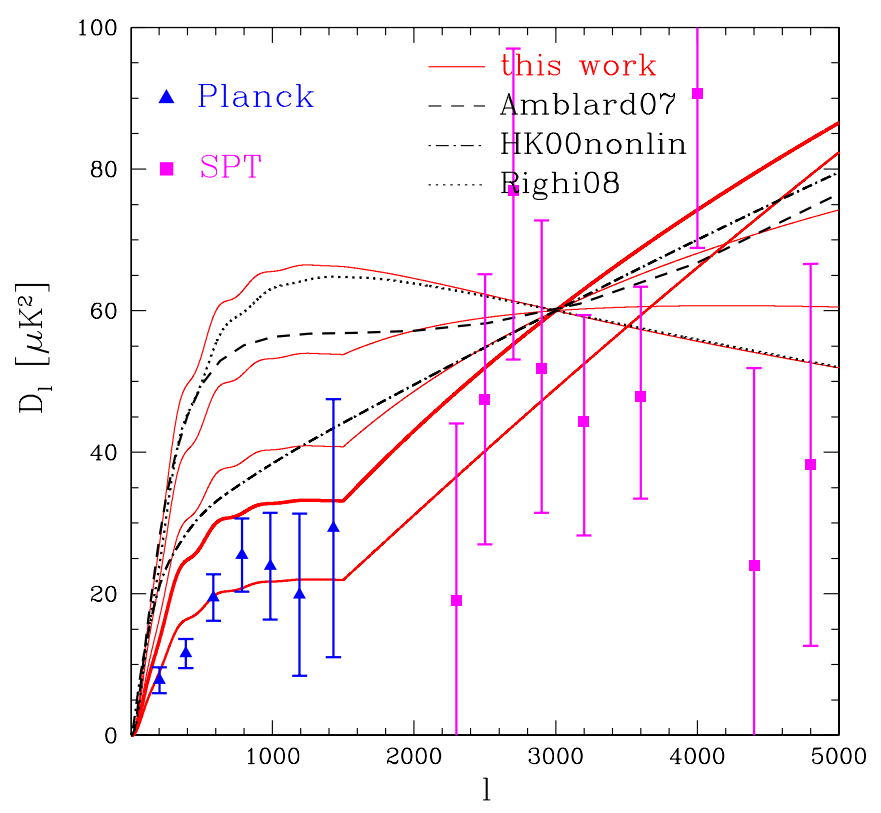

Figure 1. Three model DSFG clustering auto-spectra at $217 \mathrm{GHz}$ (black), and approximations to them with our parameterized model (solid, red), all normalized (with one exception) at $\ell=3000$. Our fiducial model is the thickest curve. Also plotted are estimates of the clustering power from Planck (Planck Collaboration 2011e) and SPT (Hall et al. 2010). For both sets of data points, we have subtracted estimates of the Poisson power from the reported total CIB power. The lowest amplitude solid (red) curve is the result of a "by-hand" adjustment of our model parameters to fit the Planck and SPT data.

(A color version of this figure is available in the online journal.)

Figure 1 that guided our understanding of the range of possible amplitudes have shapes that are inconsistent with the combined Planck and SPT data. That range of possible amplitudes is now given by the Planck CIB power spectrum measurement uncertainty.

\subsubsection{Polarization}

We expect polarized emission from the sources we consider to be very small and uncorrelated from source to source. For a collection of sources with polarization fraction $f$, contributing a Poisson temperature power spectrum of $C_{\ell}^{\mathrm{TT}, P}$, we have

$$
C_{\ell}^{\mathrm{EE}}=C_{\ell}^{\mathrm{BB}}=f^{2} C_{\ell}^{\mathrm{TT}, P} \quad C_{\ell}^{\mathrm{TE}}=f C_{\ell}^{\mathrm{TT}, P} .
$$

We parameterize both radio source and DSFG contributions with the above forms, with $f=f_{D}$ for DSFGs and $f=f_{R}$ for radio sources.

\subsection{Thermal SZ Effect}

The thermal SZ effect is a distortion of the CMB caused by inverse Compton scattering of CMB photons off electrons in the high temperature plasma within galaxy clusters. To first order, the temperature change of the CMB at frequency $v$ is given by $\Delta T / T_{\mathrm{CMB}}\left(x_{v}\right)=f\left(x_{v}\right) y$, where $f\left(x_{v}\right)=x_{v}\left(\operatorname{coth}\left(x_{v} / 2\right)-4\right)$, $x_{v}=h v / k_{B} T_{\mathrm{CMB}}$, and $y$ is the dimensionless Compton- $y$ parameter

$$
y=\left(\frac{k_{B} \sigma_{T}}{m_{e} c^{2}}\right) \int n_{e}(l) T_{e}(l) d l,
$$

where the integral is along the line of sight. $T_{\mathrm{CMB}}$ is the $\mathrm{CMB}$ temperature, and $n_{e}$ and $T_{e}$ are the number density and electron temperature of the ICM, respectively.
The thermal SZ power spectrum can be calculated by simply summing up the squared, Fourier-space SZ profiles, $\tilde{y}$, of all clusters:

$$
C_{\ell}^{\mathrm{tSZ}}=f\left(x_{v}\right)^{2} \int d z \frac{d V}{d z} \int d \ln M \frac{d n(M, z)}{d \ln M} \tilde{y}^{2}(M, z, \ell),
$$

where $\mathrm{V}(\mathrm{z})$ is the comoving volume per steradian and $n(M, z)$ is the number density of objects of mass $M$ at redshift $z$. For the latter we use the fitting function of Tinker et al. (2008). $y(M, z, r)$ is the projected radial SZ profile for a cluster of mass $M$ and redshift $z$. Note that this calculation assumes that halos are not spatially correlated; Komatsu \& Kitayama (1999) demonstrated that for $\ell>1000$ the two-halo (or clustered) contribution to the tSZ power spectrum is several orders of magnitude smaller than the Poisson contribution given by Equation (8).

To calculate the thermal SZ signal we adopt the analytic intracluster gas model presented in Shaw et al. (2010). This model provides a prescription for calculating the Compton- $y$ (or equivalently, thermal pressure) profiles of hot gas in groups and clusters. The model assumes that gas resides in hydrostatic equilibrium in the potential well of dark matter halos with a polytropic equation of state. The dark matter potential is modeled by a Navarro-Frenk-White profile (Navarro et al. 1997) using the halo-mass-concentration relation of Duffy et al. (2008). The model includes parameters to account for gas heating via energy feedback (from AGNs or supernovae) plus dynamic heating via mergers. The stellar component of the baryon fraction in groups/clusters is determined using the stellar-mass-fractiontotal-mass relation observed by Giodini et al. (2009). A radially dependent non-thermal pressure component of the gas is incorporated by calibrating off the non-thermal pressure profiles measured in hydrodynamic simulations (Lau et al. 2009). In total the model has four free parameters relating to astrophysical processes in groups and clusters. Shaw et al. (2010) explored the range in which these parameters reproduce radial profiles and scaling relations derived from X-ray observations of nearby groups and clusters.

To allow the astrophysical uncertainty to be marginalized over quickly in our Markov Chain Monte Carlo (MCMC) chains, we perform a principal component analysis (PCA) described in Appendix A. A suite of 10,000 simulated power spectra were created, each time randomly sampling from the input astrophysical parameter distribution (with the cosmological parameters fixed to their fiducial values described in Section 3). We find that two principal components are sufficient to achieve $1 \%$ accuracy out to $\ell=10,000$ on the model power spectra.

In Figure 2 we plot the thermal SZ power spectrum predicted by a number of recent simulations (black lines) as well as a fit to each with our PCA model (red lines). The dotted line represents the thermal SZ power spectrum measured from the Mare Nostrum simulation - a non-radiative simulation run using the smoothed particle hydrodynamics code, Gadget-2. The black solid line shows the results of the non-radiative simulation of Battaglia et al. (2010) and the black dashed line the results of a rerun of this simulation including radiative cooling, star formation, and energy feedback. The dot-dashed line shows the "standard" tSZ model from the simulations of Trac et al. (2010). The thickest red line represents our fiducial thermal SZ model in this work. The blue point with error bars show the recent SPT constraint on the amplitude of thermal SZ power at $\ell=3000$. All models are plotted at $146 \mathrm{GHz}$ and have been scaled to our fiducial cosmology. 


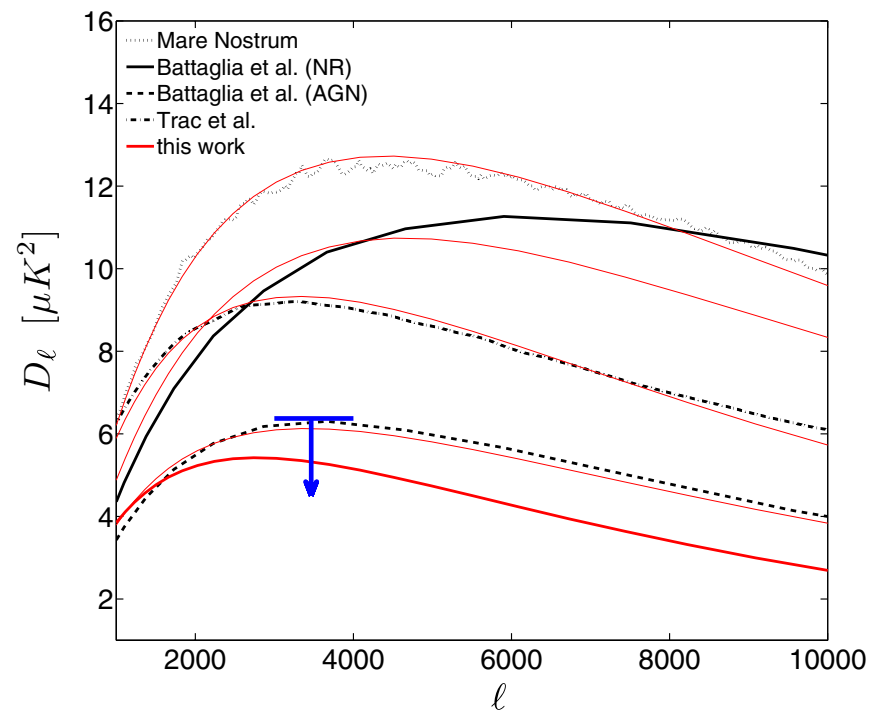

Figure 2. Comparison between recent models and simulations of the tSZ effect (black lines) and fits of our PCA model to each (thin red lines). The thickest red line shows the fiducial tSZ power spectrum used in this work. All results are plotted at $146 \mathrm{GHz}$ and are scaled to $\sigma_{8}=0.8$. The blue arrow shows the SPT $95 \%$ confidence upper limit on thermal SZ power at $\ell=3000$ (Shirokoff et al. 2010).

(A color version of this figure is available in the online journal.)

Our PCA model can accurately reproduce all the simulations in Figure 2 other than the non-radiative simulation of Battaglia et al. (2010), which peaks at much smaller angular scales than the other simulations. We note that the Shaw et al. (2010) model inherently assumes that some fraction of cluster gas has been converted to stars, whereas this simulation did not include these processes. Turning off star formation produces a power spectrum that peaks at smaller scales. In Section 7.2 we investigate the bias on measured cosmological parameters when the Battaglia et al. (2010) non-radiative template is used for the tSZ signal. We find that the PCA will adapt sufficiently to prevent a bias in the measured cosmological parameters.

The final step is to determine the cosmological scaling of the power spectrum of our fiducial model so that the amplitude can be scaled accordingly in our analysis. We find that the tSZ power spectrum is principally sensitive to $\Omega_{m}, \Omega_{b}, n_{s}$, and $\sigma_{8}$, with a particularly strong dependence on the latter. To determine the scaling we simply evaluate the Shaw et al. (2010) model varying each cosmological parameter in the range $\pm 25 \%$ of its fiducial value while holding the other three fixed (at their fiducial value). We then fit to the resulting power spectra, with our results summarized in Table 1.

\subsection{Kinetic SZ Effect}

The kinetic SZ effect is a temperature anisotropy that arises from the Compton scattering of CMB photons off of electrons that have been given a line-of-sight peculiar velocity by density inhomogeneities in the matter field. We break up the $\mathrm{kSZ}$ into contributions from the post-reionization period and from a period of inhomogeneous "patchy" reionization.

\subsubsection{Ostriker-Vishniac Effect}

When the density fluctuations which source electron velocities are in the linear regime the effect is known as the OV effect, as derived in Ostriker \& Vishniac (1986) and Vishniac (1987).
Table 1

SZ Cosmological Scaling

\begin{tabular}{|c|c|c|c|c|c|c|}
\hline$\ell$ & $\begin{array}{c}A_{\mathrm{OV}} \\
\left(\mu \mathrm{K}^{2}\right)\end{array}$ & $\begin{array}{c}n_{s} \\
0.96\end{array}$ & $\begin{array}{c}\Omega_{b} \\
0.045\end{array}$ & $\begin{array}{c}\Omega_{c} \\
0.22\end{array}$ & $\begin{array}{c}\sigma_{8} \\
0.8\end{array}$ & $\begin{array}{c}\tau \\
0.09\end{array}$ \\
\hline 500 & 1.18 & -1.44 & 1.83 & -1.06 & 4.36 & 0.25 \\
\hline 1000 & 1.81 & -1.36 & 1.91 & -1.13 & 4.82 & 0.24 \\
\hline 2000 & 2.64 & -0.94 & 1.96 & -1.12 & 5.26 & 0.22 \\
\hline 3000 & 3.06 & -0.45 & 1.94 & -1.03 & 5.38 & 0.20 \\
\hline 4000 & 3.33 & -0.22 & 1.96 & -1.04 & 5.54 & 0.17 \\
\hline 5000 & 3.53 & -0.03 & 1.98 & -1.05 & 5.66 & 0.15 \\
\hline 6000 & 3.67 & 0.13 & 2.00 & -1.06 & 5.75 & 0.13 \\
\hline 7000 & 3.78 & 0.27 & 2.01 & -1.07 & 5.83 & 0.12 \\
\hline 8000 & 3.87 & 0.38 & 2.02 & -1.08 & 5.89 & 0.10 \\
\hline 9000 & 3.94 & 0.49 & 2.03 & -1.09 & 5.94 & 0.09 \\
\hline 10000 & 4.00 & 0.58 & 2.04 & -1.10 & 5.99 & 0.08 \\
\hline \multirow[t]{2}{*}{$\ell$} & $A_{\mathrm{tSZ}}$ & $n_{s}$ & $\Omega_{b}$ & $\Omega_{m}$ & $\sigma_{8}$ & $h$ \\
\hline & $\left(\mu \mathrm{K}^{2}\right)$ & 0.96 & 0.045 & 0.265 & 0.8 & 0.71 \\
\hline 500 & 2.06 & -1.01 & 2.41 & 0.69 & 8.57 & 1.30 \\
\hline 1000 & 3.59 & -0.75 & 2.45 & 0.63 & 8.49 & 1.43 \\
\hline 2000 & 4.86 & -0.36 & 2.52 & 0.53 & 8.40 & 1.65 \\
\hline 3000 & 5.04 & -0.08 & 2.57 & 0.48 & 8.36 & 1.73 \\
\hline 4000 & 4.82 & 0.15 & 2.62 & 0.44 & 8.34 & 1.88 \\
\hline 5000 & 4.44 & 0.31 & 2.66 & 0.42 & 8.33 & 2.03 \\
\hline 6000 & 4.03 & 0.49 & 2.70 & 0.39 & 8.32 & 2.13 \\
\hline 7000 & 3.62 & 0.58 & 2.73 & 0.38 & 8.32 & 2.18 \\
\hline 8000 & 3.24 & 0.77 & 2.78 & 0.36 & 8.32 & 2.27 \\
\hline 9000 & 2.89 & 0.87 & 2.80 & 0.35 & 8.33 & 2.32 \\
\hline 10000 & 2.59 & 0.96 & 2.83 & 0.34 & 8.33 & 2.37 \\
\hline
\end{tabular}

Notes. The $\ell$-dependent power-law cosmological scalings for the Ostriker-Vishniac effect and the thermal SZ effect. The numbers immediately below the cosmological parameters are the pivot points for the power law, and the numbers in the table are the power-law indices. For example, the top row says that for the OV effect, $D_{500}=1.18 \mu \mathrm{K}^{2}\left(n_{s} / 0.96\right)^{-1.44}\left(\Omega_{b} / 0.045\right)^{1.83}$.

The post-reionization $\mathrm{kSZ}$ effect can then be modeled as the nonlinear extension of the OV effect as described below.

We follow the analytic prescription given in $\mathrm{Hu}$ (2000) which describes the angular power spectrum of the linear Vishniac effect as

$$
C_{\ell}=\frac{\pi^{2}}{2 \ell^{5}} \int d \chi D_{A}^{3}\left(g \frac{\dot{G}}{G}\right)^{2} \Delta_{\delta_{b}}^{4} I_{V},
$$

where $\chi$ is the conformal time, $G$ is the cosmological growth function, $D_{A}$ is the comoving angular diameter distance, $g$ is the visibility function, $\Delta_{\delta_{b}}^{2}$ is the linear theory baryon density power spectrum, and $I_{V}$ represents the mode coupling of the linear density and velocity fields:

$$
I_{V}=\int_{0}^{\infty} d y_{1} \int_{-1}^{1} d \mu \frac{\left(1-\mu^{2}\right)\left(1-2 \mu y_{1}\right)}{y_{1}^{3} y_{2}^{5}} \frac{\Delta_{\delta_{b}}^{2}\left(k y_{1}\right)}{\Delta_{\delta_{b}}^{2}(k)} \frac{\Delta_{\delta_{b}}^{2}\left(k y_{2}\right)}{\Delta_{\delta_{b}}^{2}(k)}
$$

with

$$
\mu=\hat{\mathbf{k}} \cdot \hat{\mathbf{k}}_{1} \quad y_{1}=k_{1} / k \quad y_{2}=k_{2} / k=\sqrt{1-2 \mu y_{1}+y_{1}^{2}} .
$$

Due to an incomplete treatment of the effects of pressure feedback from baryons we slightly overpredict the power on very small scales. As described in $\mathrm{Hu}$ (2000), in this formulation we can consider the $\mathrm{kSZ}$ effect to be the nonlinear extension of the linear Vishniac effect. This approximation requires replacing the linear density power spectrum in Equation (9) with its nonlinear extension while leaving the contribution from the 


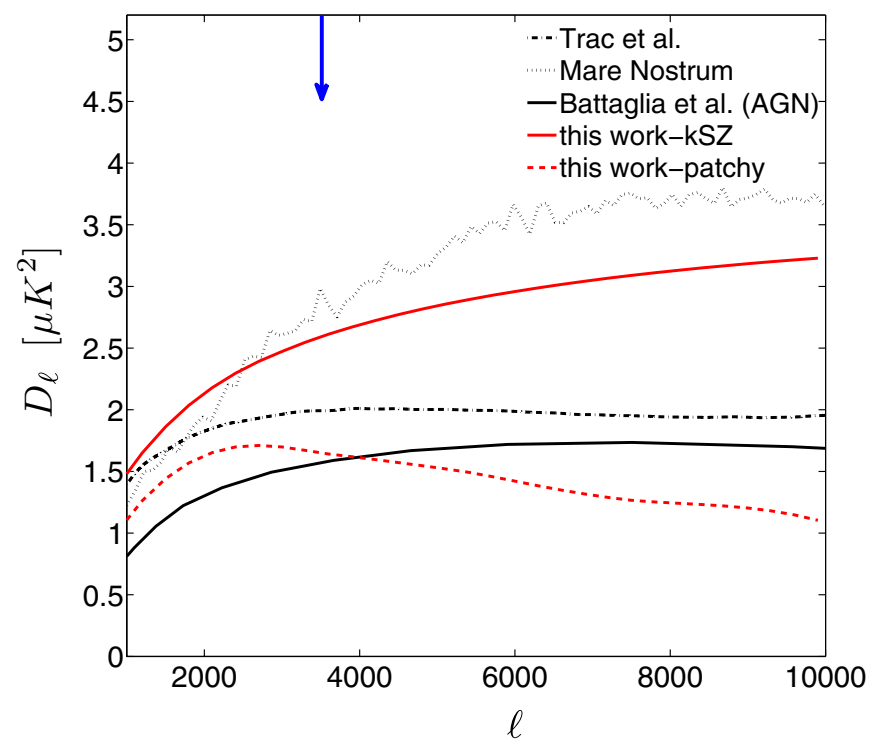

Figure 3. Comparison between recent simulations of the kSZ effect (black lines) and our model. We consider contributions from the post-reionization kSZ effect (solid red line) and from patchy reionization (dashed red line). Note that the simulations plotted here assume homogeneous reionization and thus do not include a patchy contribution. The SPT $95 \%$ confidence upper limit for the $\mathrm{kSZ}$ power at $\ell=3000$ is $6.5 \mu \mathrm{K}^{2}$.

(A color version of this figure is available in the online journal.)

velocity power spectrum unchanged:

$$
C_{\ell}^{\mathrm{kSZ}, \mathrm{OV}}=\frac{\pi^{2}}{2 \ell^{5}} \int d \chi D_{A}^{3}\left(g \frac{\dot{G}}{G}\right)^{2} \Delta_{\delta_{b}}^{2(\mathrm{NL})} \Delta_{\delta_{b}}^{2} I_{V} .
$$

For the nonlinear power spectra (NL) we utilize the HALOFIT (Smith et al. 2003) model. In this calculation of the kSZ effect we assume that the nonlinear density fluctuations are uncorrelated with the bulk velocity field in which they lie. Zhang et al. (2004) argue that this approximation may not hold in highly nonlinear regimes where contributions from the curl of the nonlinear velocity field may become important; however, we neglect these corrections here.

As in the previous section, we find a power-law approximation for estimating the $\mathrm{kSZ}$ power as a function of cosmological parameters. The kSZ angular power spectrum was calculated under the full analytic formulation for a large suite of seven-year Wilkinson Microwave Anistropy Probe (WMAP7)allowed LCDM cosmologies. An MCMC was then performed in the six-dimensional fitting-function parameter space and bestfit marginalized values were found and are listed in Table 1.

In Figure 3 we compare our calculation of the $\mathrm{kSZ}$ power spectrum (solid red line) with that measured from recent simulations (black lines). As in Figure 2, we plot the power spectrum predicted by the Mare Nostrum simulation (dotted), the "standard" model of Trac et al. (2010; dot-dashed) and the non-radiative hydrodynamic simulations of Battaglia et al. (2010; solid). The red dashed line shows our model for the contribution to the $\mathrm{kSZ}$ signal from inhomogeneous reionization (not included in the other lines), as described in the following section.

\subsubsection{Patchy Reionization}

We also consider the contribution to the kSZ power from inhomogeneous reionization (Gruzinov \& Hu 1998; Knox et al. 1998; Hu 2000; Zahn et al. 2005; McQuinn et al. 2005; Iliev et al. 2007). Simulations and analytic models of H II bubble formation both indicate that the first galaxies and quasars were highly clustered and led to gradual reionization in "bubbles" that quickly grew to sizes of several Mpc (Zahn et al. 2010).

In our estimates we use the analytic Monte Carlo "FFRT" model of Zahn et al. (2010). It has been shown to agree well with the most sophisticated radiative transfer simulations on scales of 100 comoving Mpc $h^{-1}$, while having the added advantage of allowing the modeling of arbitrarily large volumes (the analytic scheme is about four orders of magnitude faster, at a given dynamic range, than radiative transfer). This is especially important for kSZ, since large-scale velocity streams lead to the bulk of the signal. Our particular template (dashed red line in Figure 3) was calculated in a $1.5 \mathrm{Gpc} h^{-1}$ cosmological volume where $x$ - and $y$-axes correspond to roughly $15 \mathrm{deg}$ on a side and $z$-axis corresponds to redshift, with a median redshift of 8 . We shift this template left-right logarithmically by a "patchy shift" parameter $R_{P}$; that is, the power spectrum for a given shift is related to the fiducial $R_{P}=1$ spectrum by

$$
C_{\ell}=C_{R_{P} \times \ell}^{\mathrm{fid}} .
$$

$R_{P}$ is to be thought of as scaling the size of the bubbles, and is, to good approximation, proportional to the duration of the patchy phase. The timing of reionization has a secondary small effect on the shape and amplitude, which we neglect here.

\section{4. $t$ SZ-DSFG Correlation}

It is reasonable to expect some correlation between the DSFG clustering and tSZ components since they both trace the same underlying dark matter distribution, with significant overlap in redshift. Simulations which associate emission with individual cluster member galaxies predict anti-correlations-DSFGs fill in SZ decrements at frequencies below $217 \mathrm{GHz}$ considered here-on the order of tens of percent, with a correlation coefficient nearly independent of scale (Sehgal et al. 2010). This effect was explored in Shirokoff et al. (2010) who found correlation consistent with zero but with significant uncertainty due to degeneracies with the tSZ and $\mathrm{kSZ}$ components.

Assuming a fixed correlation $r_{\mathrm{tSZ}, C}$, the total power spectrum is not simply the sum of the tSZ and DSFG clustering terms, but must also include a term given by

$$
C_{\ell, v v^{\prime}}=r_{\mathrm{tSZ}, C}\left(\sqrt{C_{\ell, v v}^{C} C_{\ell, v^{\prime} v^{\prime}}^{\mathrm{tSZ}}}+\sqrt{C_{\ell, v v}^{\mathrm{tSZ}} C_{\ell, v^{\prime} v^{\prime}}^{C}}\right) .
$$

Note that this effect can be larger than either component individually in cross spectra between frequencies in which each component is large. For example, even with only moderate levels of correlation, the $217 \times 70 \mathrm{GHz}$ foreground contribution would be dominated by this correction because of the large DSFG power at $217 \mathrm{GHz}$ and $\mathrm{tSZ}$ power at $70 \mathrm{GHz}$.

\subsection{Galactic Foregrounds}

Though galactic foreground cleaning represents a key challenge for Planck, the aim of this paper is to understand the impact of the extragalactic foregrounds rather than to provide the most accurate Planck forecast possible. For treatments of galactic foregrounds see, for example, Tegmark et al. (2000) and Gold et al. (2011). The impact of ignoring the galactic foregrounds on the temperature power spectrum is minimal provided sufficiently conservative masking and template cleaning. Our neglect of galactic foregrounds will be a bad approximation at low- $\ell$ polarization and leaves our forecasts for $r$ and $\tau$ overly optimistic; we do not report these forecasts. 
Table 2

Summary of Model Parameters

\begin{tabular}{|c|c|c|c|}
\hline Parameter & Fiducial Value & $\begin{array}{l}\text { Current Constraints } \\
(1 \sigma)\end{array}$ & Definition \\
\hline \multicolumn{4}{|c|}{ Cosmological } \\
\hline$\Omega_{b} h^{2}$ & .022565 & .00073 & Baryon density \\
\hline$\Omega_{c} h^{2}$ & .10709 & .0063 & Cold dark matter density \\
\hline$\Theta$ & .010376 & .000029 & Angular size of the sound horizon at last scattering \\
\hline$\tau$ & .0799 & .015 & Optical depth to reionization \\
\hline$w$ & -1 & .13 & Dark energy equation of state parameter \\
\hline$n_{s}$ & .9669 & .014 & Scalar spectral index \\
\hline $\ln \left(10^{10} A_{s}\right)$ & 3.1462 & .045 & Scalar amplitude \\
\hline$r$ & .13 & $<.36(95 \%)$ & Tensor-to-scalar ratio \\
\hline \multicolumn{4}{|c|}{ Dusty Poisson } \\
\hline$\alpha_{D}$ & 3.8 & 0.35 & Spectral index \\
\hline$\sigma_{D}$ & .4 & & Spectral index intrinsic spread \\
\hline $\mathcal{D}_{D}$ & $5.9 \mu \mathrm{K}^{2}$ & 0.8 & Amplitude at $\ell=3000, v=143 \mathrm{GHz}$ \\
\hline$f_{D}$ & .01 & & Dusty polarization fraction \\
\hline \multicolumn{4}{|c|}{ Radio Poisson } \\
\hline$\alpha_{R}$ & -.5 & 0.1 & Spectral index \\
\hline$\sigma_{R}$ & .1 & $<0.6(95 \%)$ & Spectral index intrinsic spread \\
\hline $\mathcal{D}_{R}$ & $53 \mu \mathrm{K}^{2}$ & $10 \mu \mathrm{K}^{2}$ & Amplitude at $\ell=3000, v=143 \mathrm{GHz}, S_{c}=330 \mathrm{mJy}$ \\
\hline$\gamma_{R}$ & -.8 & 0.1 & Brightness function power-law index \\
\hline$f_{R}$ & .05 & & Polarization fraction \\
\hline \multicolumn{4}{|c|}{ Dusty Clustered } \\
\hline$\alpha_{C}$ & 3.8 & 0.4 & Spectral index \\
\hline $\mathcal{D}_{C}$ & $3.9 \mu \mathrm{K}^{2}$ & $1.2 \mu \mathrm{K}^{2}$ & Amplitude at $\ell=3000, v=143 \mathrm{GHz}$ \\
\hline$n_{C}$ & 1 & & Nonlinear tilt \\
\hline \multicolumn{4}{|l|}{ SZ Effects } \\
\hline $\mathcal{D}_{\mathrm{tSZ}}$ & $4.3 \mu \mathrm{K}^{2}$ & $<6.8 \mu \mathrm{K}^{2}(95 \%)$ & tSZ amplitude at $\ell=3000, v=143 \mathrm{GHz}$ \\
\hline $\mathcal{D}_{\mathrm{kSZ}, \mathrm{OV}}$ & $2.7 \mu \mathrm{K}^{2}$ & $<6.5 \mu \mathrm{K}^{2}(95 \%)$ & OV amplitude at $\ell=3000$ \\
\hline $\mathcal{D}_{\mathrm{kSZ}, P}$ & $1.5 \mu \mathrm{K}^{2}$ & $<6.5 \mu \mathrm{K}^{2}(95 \%)$ & Patchy amplitude at $\ell=3000$ \\
\hline$R_{P}$ & 1 & & Patchy shift \\
\hline \multicolumn{4}{|c|}{ Correlations } \\
\hline$r_{\mathrm{tSZ}, C}$ & 0 & & Correlation between $\mathrm{tSZ}$ and DSFGs at $\ell=3000$ \\
\hline
\end{tabular}

Notes. A summary of the parameters in our model. The fiducial values generate our simulated data. The current constraints column gives the $1 \sigma$ constraints on our model given WMAP power spectra and radio source counts, SPT power spectra and radio/DSFG source counts, and ACT power spectra. Note that due to the process by which the fiducial values were chosen (Section 3) they are not necessarily the most likely values given current data; they are, however, totally consistent with the most likely value to within $1 \sigma$.

\section{6. $C M B$}

For the primary CMB signal itself, we use an eight-parameter model which includes the baryon density $\Omega_{b} h^{2}$, the density of cold dark matter $\Omega_{c} h^{2}$, the optical depth to recombination $\tau$, the angular size of the sound horizon at last scattering $\Theta$, the amplitude of the primordial density fluctuations $\ln \left[10^{10} A_{s}\right]$, the scalar spectral index $n_{s}$, the dark energy equation of state parameter $w$, and the tensor-to-scalar ratio $r$. Freeing $w$ opens up the "geometric degeneracy" which is typically broken by adding an external data set such as supernovae data. Rather than doing this, we simply put a $\pm 0.3 \mathrm{G}$ prior on $w$ to reasonably constrain the chain while allowing it to explore the parameter space.

Because we are interested in the simplest description of how the foregrounds affect cosmological parameters, we do not consider extensions to our model such as a running spectral index, non-flat universes, non-standard effective number of neutrino species, or a difference in primordial helium from standard big bang nucleosynthesis. Due to the small angular scales where they affect the CMB anisotropy, it is possible that such parameters could be even more degenerate with the foregrounds than the "vanilla" set we consider.
For quick and highly accurate $\mathrm{CMB}$ calculations during our MCMC chains, we use a PICO (Fendt \& Wandelt 2007) interpolation of a training set generated by CAMB (Lewis et al. 2000). PICO was trained using the 2008 June version of CAMB which uses a now-outdated recombination code. Though we use the older code, we do it in a self-consistent manner and do not expect any impact on our forecasting. Additionally, the training set includes the option of a nonlinear lensing contribution described in Challinor \& Lewis (2005) which we use.

\section{FIDUCIAL MODEL AND CURRENT CONSTRAINTS}

For our forecasting, we create simulated power spectra (henceforth the "simulated data") using the model described in the previous sections. We pick one single set of model parameter values, called the "fiducial values" or the "fiducial model" in general, which is the baseline for the different cases of simulated data which we consider. The model used to analyze the simulated data, which generally contains small changes relative to the fiducial model, will be called the "analysis model."

In Table 2 we summarize all of the parameters in our model, the naming convention, and their fiducial values. The fiducial values are chosen to be consistent with current cosmological 
constrains from WMAP7 (Komatsu et al. 2010), and with constraints on the foreground components from ground-based data such as SPT and ACT. In the following paragraphs, we describe the method used to arrive at our fiducial model.

Because the expected SZ power depends strongly on cosmology, special care was taken so that our fiducial SZ power and cosmology agree. To achieve this, we use the constraint from Lueker et al. (2010) on the linear combination $\mathcal{D}_{\mathrm{tSZ}}+.46 \times \mathcal{D}_{\mathrm{kSZ}}=$ $4.2 \pm 1.5 \mu \mathrm{K}^{2}$ (at $\ell=3000$ and $153 \mathrm{GHz}$ ) along with the cosmological scalings in Table 1 . We then importance sample the WMAP7 $\Lambda$ CDM+TENS ${ }^{11}$ chain by calculating at each step the expected SZ (kinetic and thermal) power assuming no theory uncertainty, then applying the prior from Lueker et al. (2010). The new best-fit point in the post-processed chain mainly shifts SZ power up relative to best-fit SPT value and $\sigma_{8}$ down relative to the best-fit WMAP7 value. All other cosmological parameters are also affected (at a smaller level), and their new mean values form our fiducial cosmology, which remains $1 \sigma$ consistent across all parameters with WMAP7.

For the radio sources, the tightest constraints on the expected Planck power come from the Vieira et al. (2010) catalog which contains sources in the decade of brightness just below the Planck flux cut. Fitting a de Zotti et al. (2005) model to the data yields the values listed in Table 2, notably radio Poisson power of $53 \mu \mathrm{K}^{2}$ at $143 \mathrm{GHz}$ assuming a $330 \mathrm{mJy}$ flux cut.

Since the DSFG Poisson contribution is nearly independent of flux cut, we expect the same Poisson power in Planck maps as in SPT maps, adjusting only for bandpass differences. We get our fiducial value for Planck Poisson power at $143 \mathrm{GHz}$ by extrapolating in frequency from the best-fit value of the SPT $150 \mathrm{GHz}$ power as given in Shirokoff et al. (2010). Our fiducial values for $\alpha_{D}$ and $\alpha_{C}$ also come from the best-fit values in Shirokoff et al. (2010). We set $\sigma_{D}$ to 0.4 following the arguments in Knox et al. (2004), although it is not yet well constrained by observations. We adopt a clustering tilt $n_{C}=1$ so that it (roughly) has the shape expected at small scales due to the observed clustering properties of high-redshift galaxies. As argued by Scott \& White (1999), the observed clustering properties of $z \sim 3$ Lyman break galaxies, namely an angular correlation function proportional to $\theta^{-0.9}$ (Giavalisco et al. 1998), correspond to $D_{\ell} \propto \ell^{1.1}$. Since we multiply the power law by the linear theory template, this is similar (at $\ell>1500)$ to the power law only $D_{\ell} \propto \ell^{0.8}$ shape used as baseline models in both Dunkley et al. (2011) and Shirokoff et al. (2010). Although ruled out by the Planck data, our fiducial model is at least closer to the measurements than all of the other models plotted in Figure 1. The agreement is sufficient for our purposes here, though we will certainly be updating our CIB modeling in the near future.

Following Battye et al. (2011) which found a mean fractional polarization of $4.5 \%$ at $86 \mathrm{GHz}$ (and varying weakly with frequency) for the WMAP point-source catalog (Wright et al. 2009), we adopt a fiducial value of $f_{R}=0.05$. For DSFGs we expect an even smaller level of average polarization fraction. Polarized dust emission arises due to alignment of grains in interstellar magnetic fields. We somewhat arbitrarily set $f_{D}=0.01$ for our fiducial model, which is consistent with the finding that, in our own Galaxy, the coherence length for magnetic fields is much smaller than the extent of the dust emission (Prunet et al. 1998).

\footnotetext{
11 Available at http://lambda.gsfc.nasa.gov/.
}

Table 3

Survey Properties

\begin{tabular}{lccl}
\hline \hline $\begin{array}{l}\text { Band } \\
(\mathrm{GHz})\end{array}$ & $\begin{array}{c}\text { T (E/B) } \\
(\mu K \text {-arcmin })\end{array}$ & $\begin{array}{c}\text { Beam } \\
(\operatorname{arcmin})\end{array}$ & Notes \\
\hline Planck & $177(253)$ & 14 & $f_{\text {sky }}=70 \%$ \\
70 & $61(98)$ & 10 & $S_{c}=330 \mathrm{mJy}$ \\
100 & $42(80)$ & 7.1 & \\
143 & $64(132)$ & 5 & \\
217 & & & $f_{\text {sky }}=100 \mathrm{deg}^{2}$ \\
\hline Ground-Deep & 53 & 1.6 & $S_{c}=6.4 \mathrm{mJy}$ \\
90 & 13 & 1.15 & \\
150 & 35 & 1.05 & $f_{\text {sky }}=1000 \mathrm{deg}^{2}$ \\
220 & & & $S_{c}=6.4 \mathrm{mJy}^{2}$ \\
\hline Ground-Wide & 53 & 1.6 & \\
90 & 18 & 1.15 & \\
150 & 80 & 1.05 & \\
220 & & & \\
\hline
\end{tabular}

Notes. Instrument properties used to generate simulated power spectra. The beam width is given as an FWHM. $S_{c}$ refers to the flux cut above which brighter sources are masked out.

Figure 4 shows the fiducial CMB and foreground contribution to Planck TT, TE, and EE power spectra (with the exception of tSZ-DSFG correlation, which is plotted at $30 \%$ rather than its fiducial value of $0 \%$ ).

\section{SURVEY PROPERTIES}

We consider simulated Planck data in the four bands between $70 \mathrm{GHz}$ and $217 \mathrm{GHz}$. These are chosen because they contain nearly all of the significant $\mathrm{CMB}$ information. Though the neglected channels place little extra constraints on the CMB, they are crucial for understanding and cleaning the foregrounds. We consider their effect implicitly by testing limits such as lowered Poisson power amplitudes, or fixed DSFG clustering shapes. Additionally, we also consider the benefit of higher resolution ground-based data, which we model after SPT $90 \mathrm{GHz}$, $150 \mathrm{GHz}$, and $220 \mathrm{GHz}$ channels. We divide the data into two fields: a $100 \mathrm{deg}^{2}$ "deep" field and a $1000 \mathrm{deg}^{2}$ "wide" field. We henceforth refer to these two data sets as Ground-deep and Ground-wide. The depths, sky coverage, and flux cuts used in our forecasting are summarized in Table 3.

Our simulated data take the form of auto and cross spectra from as many bands as are present for a given patch of sky. The four Planck frequency channels form $10 \mathrm{TT}, \mathrm{EE}$, and BB and 16 TE power spectra, with an additional 18 TT power spectra from the three extra frequencies in regions of Ground-overlap. ${ }^{12}$ We do not assume overlap between Ground-deep and -wide, nor do we form cross spectra between Ground temperature and Planck polarization as these are expected to be a very small contribution to the $\mathrm{CMB}$ and foreground information. Planck $\mathrm{BB}$ polarization is also ignored except in one test case where we find its impact is minimal on our cosmological parameterization.

We simulate power spectrum assuming a uniform masking threshold across the sky. The only exception is in the case of Planck and Ground-overlap. For such patches of sky, we assume Planck maps can be masked using a point-source mask from the higher resolution Ground data. Thus, for the overlap areas, even the Planck auto spectra will have greatly reduced radio Poisson power. 12 In general $N$ frequency channels can be used to create $N(N+1) / 2$ power
spectra of type TT, EE, and $\mathrm{BB}$, and $N^{2}$ of type TE. 

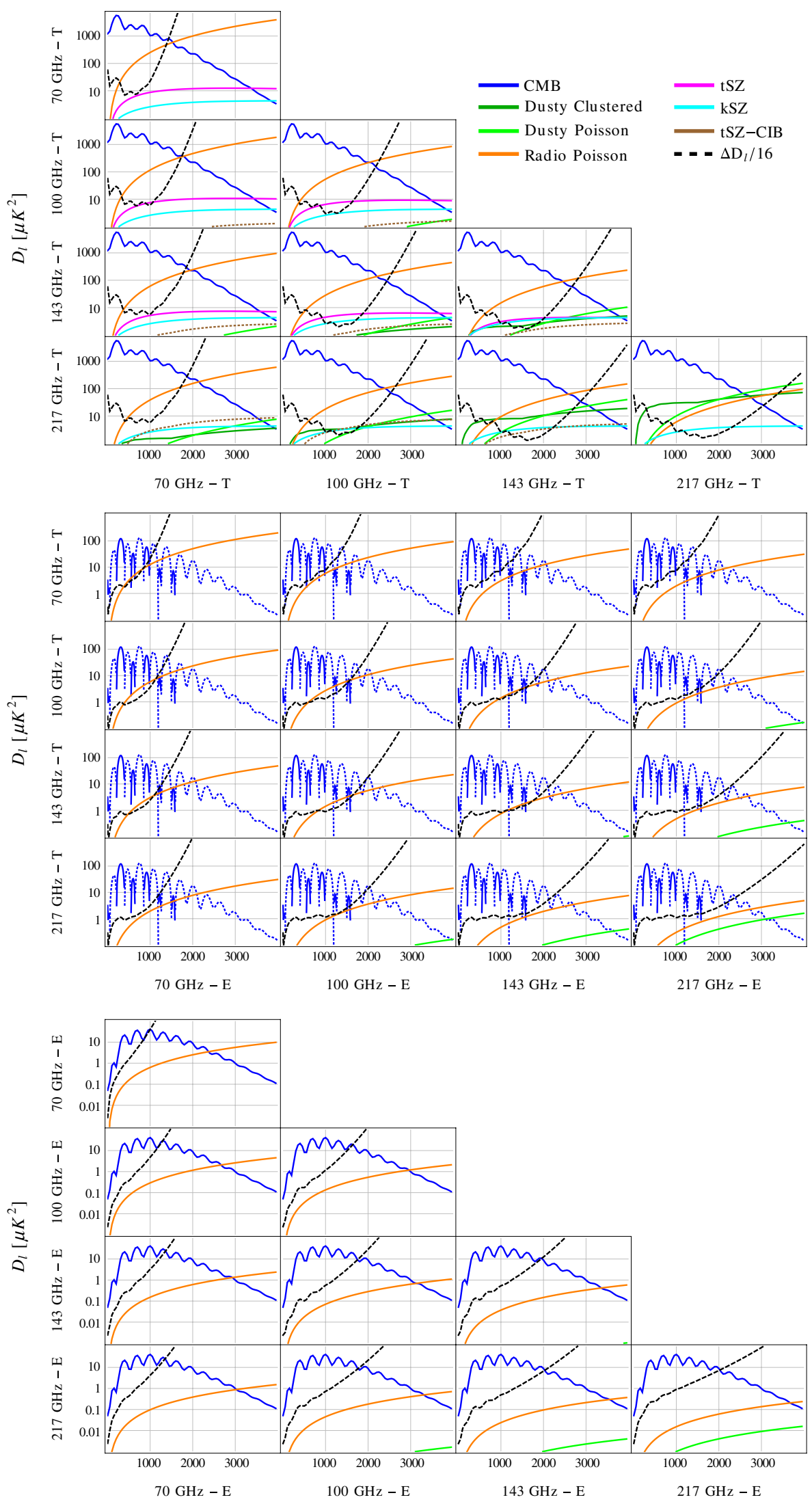

Figure 4. All 36 power spectra which can be formed from Planck $70 \mathrm{GHz}-217 \mathrm{GHz}$ temperature and E-mode polarization, and the prediction of our fiducial model for the CMB and foreground power in each of them (with the exception of the tSZ-DSFG correlation, which is shown at $30 \%$ instead of its fiducial value of 0\%). The black dashed line shows the errors bars for $\ell$-bins of width $\Delta \ell=256$. Dotted lines indicate negative power.

(A color version of this figure is available in the online journal.) 
The non-zero width of frequency bandpasses creates a different effective frequency for each component in each band. For components with uncertain spectral shapes, the variation in effective frequency leads to percent level corrections which can be neglected. In this paper, values quoted from ground-based experiments are normalized at, and explicitly cite, the corresponding effective frequency. For Planck, it is sufficient for our forecasting purposes to ignore this and use the nominal band centers for all components.

\section{FORECASTING METHODOLOGY}

The analysis of the simulated data assumes perfectly known Gaussian beams, no calibration uncertainty, isotropic noise, and ignores the effects of mode-mode coupling on the cut sky. While these assumptions are not sufficient for modeling real data, we expect them to be adequate for our purpose of modeling the extragalactic foregrounds, and understanding their importance on cosmological parameter biases and statistical errors.

Under these assumptions, the so-called "pseudo power spectrum" which includes both signal and noise is given by

$$
C_{i j, \ell}=C_{i j, \ell}^{S}+\delta_{i j} w^{-1} \exp \left(\ell^{2} \sigma_{b}^{2}\right) .
$$

Here $i$ and $j$ each label one of the maps, and the noise is parameterized by the weight per solid angle $w$, and the beam width in radians $\sigma_{b}$. Assuming the Gaussianity (the validity of which we discuss in Section 5.1), the covariance on our estimate of the signal power spectra is

$$
\begin{aligned}
\Sigma_{(i j)(k l)} & \equiv\left\langle\left(\widehat{C}_{i j}^{S}-C_{i j}^{S}\right)\left(\widehat{C}_{k l}^{S}-C_{k l}^{S}\right)\right\rangle \\
& =\frac{1}{(2 l+1) f_{\text {sky }}}\left(C_{i l} C_{j k}+C_{i k} C_{j l}\right),
\end{aligned}
$$

where we have suppressed the $\ell$ dependence for notational simplicity. We use the covariance to form the likelihood as a function of parameters $\theta$ :

$$
-2 \ln \mathcal{L}(\theta)=\left[C_{i j}^{S}(\theta)-\widehat{C}_{i j}^{S}\right] \Sigma_{(i j)(k l)}^{-1}\left[C_{k l}^{S}(\theta)-\widehat{C}_{k l}^{S}\right] .
$$

Note that we have neglected the normalization term since it does not vary with $\theta$.

Our simulated data are the mean expected power spectra; i.e., they do not include a sample of the errors from the bandpower covariance matrix. Leaving out these fluctuations has the benefit of making the best-fit $\chi^{2}$ equal to exactly 0 (as long as our analysis model and simulation model are the same) and has no affect on our forecasting abilities.

Tests we performed showed that a Gaussian propagation of uncertainty from the $C_{\ell}$ 's to the model parameters can be insufficiently accurate due to the highly non-Gaussian shape of the foreground parameter posterior likelihoods. Instead, we run a full MCMC analysis using a custom multi-frequency extension to CosmoMC (Lewis \& Bridle 2002). The code for this extension along with chains for the results quoted in this paper are available online. ${ }^{13}$

\subsection{Non-Gaussianity}

In the previous section, we make assumptions that the signal and noise are a Gaussian random field (Equation (16)) and that the likelihood itself is Gaussian (Equation (17)). The latter is a

\footnotetext{
13 http://student.physics.ucdavis.edu/ millea/data/millea2011
}

good approximation for examining the foregrounds since they are relevant at high- $\ell$ where averaging over many $a_{l m}$ 's drives the likelihood to Gaussianity. The former, however, can be a very bad approximation for the radio/dusty Poisson and tSZ (which is just the one-halo term) since they are sourced by Poisson number fluctuations. In general, we expect the tSZ to suffer most from non-Gaussian effects since the fluctuation power is most heavily weighted towards a small number of bright objects; the dusty Poisson component by contrast receives most of the power from well below the flux-cut where there are many sources.

Non-Gaussianity can impact our likelihood both by producing long tails in the $C_{\ell}$ probability distribution and by the $C_{\ell}$ variance differing from that expected from sample variance. The first problem is alleviated by large sky coverage. We can use the tSZ as a worst-case scenario, where calculations by Zhang \& Sheth (2007) and simulations by Peel et al. (2009) suggest that for $1000 \mathrm{deg}^{2}$ one would expect a skewness of less than 0.1 in the distribution of $C_{\ell}$ 's. The second problem, however, does not average away with $f_{\text {sky }}$, and notably becomes worse when binning in $\ell$. The full expression for the $C_{\ell}$ covariance is

$$
\left\langle\Delta C_{\ell} \Delta C_{\ell^{\prime}}\right\rangle=f_{\text {sky }}^{-1}\left\{\frac{2\left\langle C_{\ell}\right\rangle^{2} \delta_{\ell \ell^{\prime}}}{(2 \ell+1) \Delta \ell}+\frac{T_{\ell \ell^{\prime}}}{4 \pi}\right\},
$$

where the first term is the sample variance for a Gaussian field, and the second is the non-Gaussian trispectrum contribution (Komatsu \& Seljak 2002). To examine the impact of ignoring the trispectrum in our analysis, we use the following method. Since binning in $\ell$ brings out the effect of a non-zero trispectrum, we consider making one giant $\ell$-bin which is inverse-variance weighted across the entire angular range. If the variance in that bin still receives a negligible contribution from the trispectrum, then we can safely ignore its effect (including $\ell$-to- $\ell$ correlations) in our more reasonably binned analysis.

The primary contribution to the $\mathrm{tSZ}$ trispectrum comes from galaxy cluster shot noise (Komatsu \& Seljak 2002). Shaw et al. (2009) calculate this contribution, and we use their trispectrum to apply the binning procedure described above. Using only Planck $143 \mathrm{GHz}$ data and forming the single $\ell$-bin, we find that the error bar is increased by $1 \%$. For Ground $150 \mathrm{GHz}$, it is increased by $30 \%$, although we expect this number to decrease for an analysis which does not ignore mode-coupling.

To calculate the radio trispectrum, we create a set of 1000 full sky radio realizations by Poisson sampling our fiducial brightness function. We find, somewhat surprisingly, that the radio power spectrum with a Planck flux cut is even more nonGaussian than the tSZ, dropping to a similar level for a Ground flux cut. Applying the binning procedure yields an error increase of $6 \%$ for a Planck flux cut and Planck $143 \mathrm{GHz}$ data, and 4\% for a Ground flux cut and Ground $150 \mathrm{GHz}$.

Given the small error increase in all cases, we conclude that it is safe to ignore the foreground non-Gaussianities in our analysis. We do note, however, the possibility that nonGaussianities could be relevant to other analyses, for example, component separation methods which produce heavily $\ell$-binned foreground-only maps, or lensing reconstructions which rely on off-diagonal correlations.

\section{COMPRESSION TO A CMB POWER SPECTRUM ESTIMATE}

Before getting to our results, it is useful to explore the foreground contamination in a more model-independent manner, motivated by two drawbacks of our procedure. First, there is 
a large amount of data one must work with-our bandpower covariance matrix at each $\ell$ is $46 \times 46$ for Planck and $64 \times 64$ for Planck+Ground. Second, 17 foreground parameters must be marginalized over, and if one wanted to examine constraints on a new cosmological model, the whole procedure would have to be repeated. Here we present a procedure for compressing all the power spectra to (1) a single CMB estimate and (2) a low dimensional parameterization of the residual foregrounds in this estimate. We describe the procedure here for temperature-only power spectra with errors that are uncorrelated from multipole to multipole. The generalization to include $\ell$-to- $\ell$ correlations and polarization is in Appendix B.

Given $N$ power spectra (for example, the $10 \mathrm{TT}$ spectra we consider for Planck), we would like to split our data up into $N-1$ linear combinations of power spectra that have no sensitivity to the $\mathrm{CMB}$ and then find the remaining linear combination that contains $\mathrm{CMB}$ and whose errors are uncorrelated with those of the $N-1$. With this split made, we can then derive our foreground constraints using the CMB-free linear combinations. Doing so means foreground constraints can be made independent of our modeling of the CMB (other than the assumed frequency dependence).

We use the $N-1 \mathrm{CMB}$-free linear combinations to find the constraints they place on our foreground model parameters via MCMC. For each point in the chain we can determine the contribution to the CMB linear combination. We sample over all of these contributions to find the mean contribution and fluctuations about that mean. We find a low-dimensional description of the fluctuations via a principal component decomposition.

\subsection{Splitting the Power Spectra into CMB-free and a CMB Estimate}

Let us begin by first considering arbitrary linear combination of the power spectra,

$$
\tilde{C}^{\mu}=\sum_{i} w_{i}^{\mu} C_{i}
$$

where $C_{i}$ are the $i=1$ to $N$ power spectra. The weightings we consider will be $\ell$-dependent; the lack of any labeling by $\ell$ is solely for notational simplicity. Here, $\mu$ is merely a label to distinguish different weightings; the $\tilde{C}^{\mu}$ are a linear combination of the old power spectra with weight $w_{i}^{\mu}$. Note that if a weighting satisfies

$$
\sum_{i} w_{i}^{\mu}=0
$$

it is not sensitive to the CMB.

We would first like to find the CMB weighting $w^{\mathrm{CMB}}$ which will be statistically orthogonal to the $N-1$ linear combinations that satisfy the CMB-free condition (Equation (20)). We would also like this weighting to be properly normalized so that

$$
\sum_{i} w_{i}^{\mathrm{CMB}}=1
$$

To satisfy the orthogonality condition it helps to work in a primed space defined by a linear transformation via

$$
w_{\alpha}^{\prime \mu}=\sum_{i} L_{\alpha i} w_{i}^{\mu},
$$

where $L$ is the Cholesky decomposition of the bandpower error covariance matrix, $\Sigma=L L^{T}$. The advantage of the primed space is that the basis vectors in the primed space correspond to power spectra whose errors are statistically orthogonal; i.e., with the weightings set so that $w_{\alpha}^{\prime \mu}=\delta_{\mu \alpha}$ (now setting $\mu=1 \ldots N$ ) the errors in the corresponding power spectra satisfy

$$
\left\langle\delta \tilde{C}^{\mu} \delta \tilde{C}^{\nu}\right\rangle=\delta_{\mu \nu}
$$

The primed weights that satisfy the CMB-free condition satisfy

$$
\sum_{\alpha, i} L_{i \alpha}^{-1} w_{\alpha}^{\prime}=0
$$

Thus any power spectrum with primed weighting proportional to

$$
w_{\alpha}^{\prime \mathrm{CMB}}=\sum_{i} L_{i \alpha}^{-1}
$$

is perpendicular to any vector satisfying the CMB-free condition, as one can easily verify. To find the CMB weighting in the unprimed space we perform the inverse transform and normalize to satisfy the normalization condition (Equation (21)):

$$
\begin{aligned}
w_{k}^{\mathrm{CMB}} & =\sum_{i, \alpha} L_{k \alpha}^{-1} L_{i, \alpha}^{-1}\left[\sum_{i, k, \alpha} L_{k \alpha}^{-1} L_{i \alpha}^{-1}\right]^{-1} \\
& =\sum_{i} \Sigma_{i k}^{-1}\left[\sum_{i, k} \Sigma_{i k}^{-1}\right]^{-1} .
\end{aligned}
$$

Note that this is the expression for inverse-variance weighting.

Our remaining task is to construct the $N-1 \mathrm{CMB}$-free weightings in a manner that leaves them all statistically orthogonal to the CMB weighting. We do so by applying the Gram-Schmidt procedure in the primed space. This gives us $N-1$ orthogonal vectors that are all orthogonal to the CMB direction as well, that we will call $v_{\alpha}^{\prime \mu}$ for $\mu=2, N$. The weightings in the unprimed space are then given by

$$
v_{i}^{\mu}=L_{i \alpha}^{-1} v_{\alpha}^{\prime \mu}
$$

We now define the matrix $W$ so that

$$
W_{1 i}=w_{i}^{\mathrm{CMB}} \quad W_{\mu i}=v_{i}^{\mu}\{\text { for } \mu=2 \ldots N\} .
$$

This matrix defines the linear combinations of the power spectra that have all the properties we desire. The first row is the optimal $\mathrm{CMB}$ weighting and subsequent rows give the $N-1 \mathrm{CMB}-$ free linear combinations. All the linear combinations are statistically orthogonal; i.e., the covariance matrix for the new power spectra, $W^{T} \Sigma W$, is diagonal. Furthermore, $W$ is non-singular so we have not lost any information through this reweighting.

\subsection{Modeling the Foreground Residuals with Principal Components}

With the weight matrix $W$ defined, we can constrain the foreground power in the $N-1 \mathrm{CMB}$-free power spectra by running an MCMC chain. Despite the large number of parameters and power spectra, this analysis is fast in practice because the foreground model consists of simple analytic forms and pre-computed templates, and does not depend on any costly Einstein-Boltzmann solver or lensing models. For the set of foreground parameters at each step in this chain, we 
calculate the corresponding foreground contribution to the CMB linear combination. These $\ell$-dependent contributions form the columns of the $Y$ matrix in a PCA (see Appendix A). Following the PCA procedure, we have a few principal components and priors on their amplitudes which must be marginalized over in a separate chain which uses only the CMB linear combination.

\subsection{Discussion of Linear Combination Analysis}

The weightings which make up the CMB and CMB-free linear combinations depend on the bandpower covariance matrix, and thus on the noise properties of the instrument, on any filtering that is performed, and on the true power spectrum on the sky. The principal components for the foreground residuals also depend on the choice of foreground model. For a Planck temperatureonly forecast and for our fiducial model, we present the results of a linear combination analysis.

In the top panel of Figure 5 we plot the weights for the $\mathrm{CMB}$ linear combination as a function of $\ell$. At high $\ell$ where the measurement is noise dominated, nearly all of the CMB information is contained in the $217 \mathrm{GHz}$ map which is the least noisy. At lower $\ell$ where we become dominated by cosmic variance, the $\mathrm{CMB}$ information comes from the channels with the lowest foreground contamination.

Given these weights, we plot in the middle panel of Figure 5 the foreground contribution to the $\mathrm{CMB}$ linear combination and the error bars on this new power spectrum. Also shown are the error bars for the $217 \mathrm{GHz}$ channel alone for comparison. The maximum improvement is at $\ell=2000$ where the error bars tighten by a factor of 1.4. We also see that the dominant contribution to the foreground power in the $\ell$ range where Planck is most sensitive to the CMB is the radio Poisson, followed by the DSFG clustering.

Finally, we perform a PCA on the foreground residuals in the $\mathrm{CMB}$ linear combination. The first several principal components are shown in the bottom panel of Figure 5. We find that all of the variation $>1 \mu \mathrm{K}^{2}$ can be described by two amplitude parameters, compared to the 14 parameters which govern these foregrounds. Another way to put this is that using the CMB-free combinations we can clean out almost $140 \mu \mathrm{K}^{2}$ of foregrounds (at $\ell=3000$ ), leaving only tens of $\mu \mathrm{K}^{2}$ of residual uncertainty, modeled with the two principal components.

\section{RESULTS}

With the model and forecasting tools in place, we are ready to present the results of our main analysis. We want to find which components can potentially cause large biases in an analysis of Planck data, so that we can model them with sufficient care. We would also like to know how much constraining power is reduced due to foreground confusion. Could significant improvements in cosmological parameter constraints be achieved by using additional data and/or modeling? To answer these questions, we run a suite of forecasting analyses aimed at singling out the effects of each foreground contribution.

The next subsections are organized as follows. First, we examine the importance of each component by turning it on or off in the analysis. For the components which we deem important, we check whether our modeling is sufficient to protect the cosmological parameters from biases, both at the Planck and Planck+Ground sensitivity levels. We then examine the degradation in statistical errors from the need to marginalize over the foregrounds, and finally we explore the impact of adding in ground-based data.

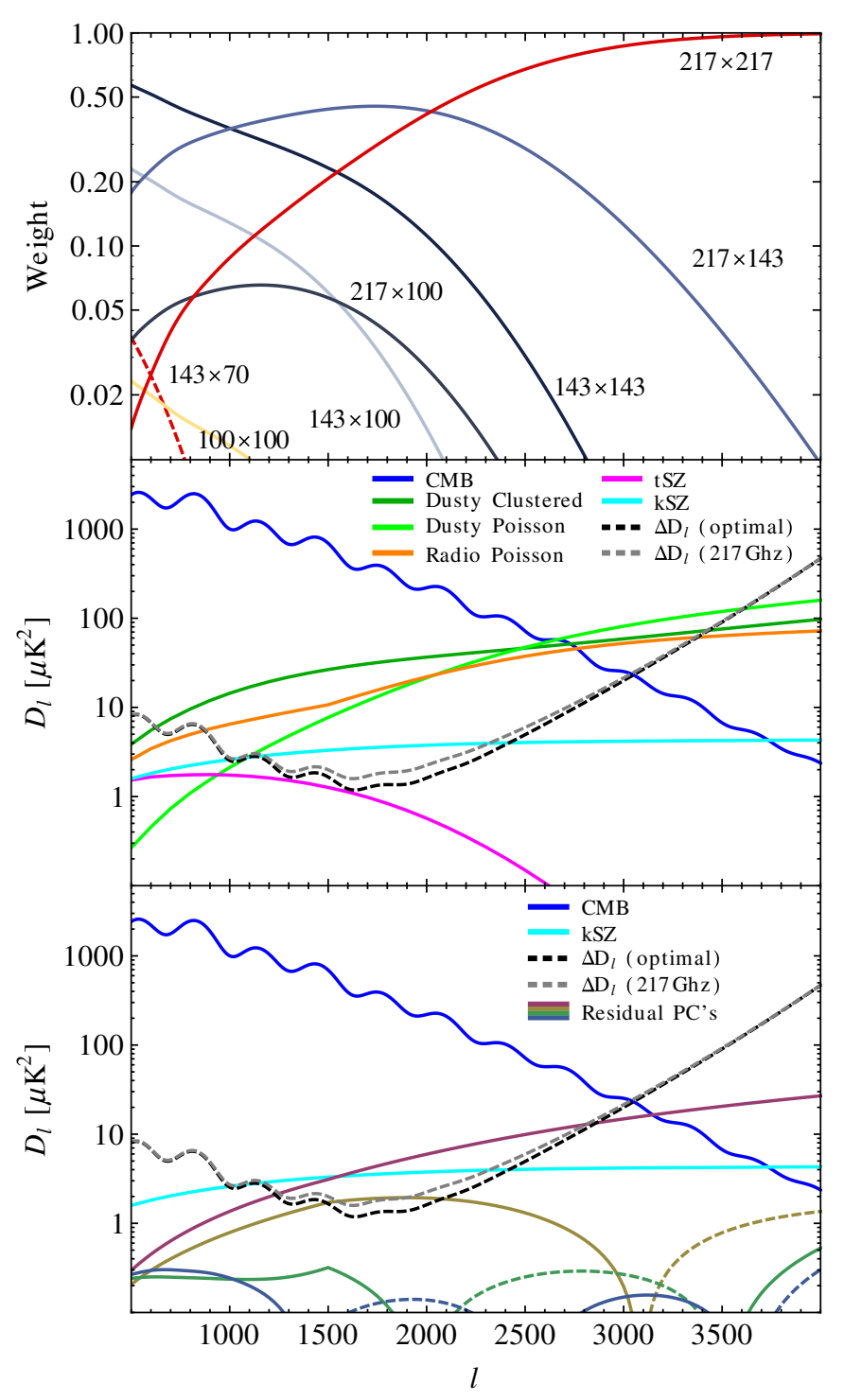

Figure 5. Top: the $\ell$-dependent weightings which form the $\mathrm{CMB}$ linear combination (Equation (29)). All possible auto/cross spectra from Planck channels in Table 3 were considered. Dashed lines indicate negative weight. Middle: the mean foreground contribution to the CMB linear combination for our fiducial model. Note, for example, that tSZ (purple) is not present at high $\ell$ because only $217 \mathrm{GHz}$ is used there. The dominant non-Poisson component for the $\ell$-range where Planck is most sensitive is the DSFG clustering. Bottom: principal components of foreground residuals (constrained by the CMB-free linear combinations) with amplitudes set to $1 \sigma$. Note that we only need two principal component amplitudes to be accurate to $>1 \mu \mathrm{K}^{2}$. (The errors in bin widths of $\Delta \ell=256$ for both the $\mathrm{CMB}$ linear combination and for $217 \mathrm{GHz}$ alone are plotted as dashed lines in the bottom two plots.)

(A color version of this figure is available in the online journal.)

\subsection{Importance of the Different Components}

Figure 6 shows the effect of removing four foreground components - the DSFG clustering, tSZ, kSZ, and tSZ-DSFG correlation-one at a time from the analysis model, while they are actually present in the simulated data at their fiducial value. We present the results by plotting likelihood contours in the $n_{s}$ and $\Omega_{c} h^{2}$ plane, since changes in those two parameters affect the primary CMB at the smallest scales and are the most susceptible to foreground biases. We also show the amplitudes of the clustering and SZ effects as their $\ell$-space shapes make them most degenerate with cosmological parameters. All of the 

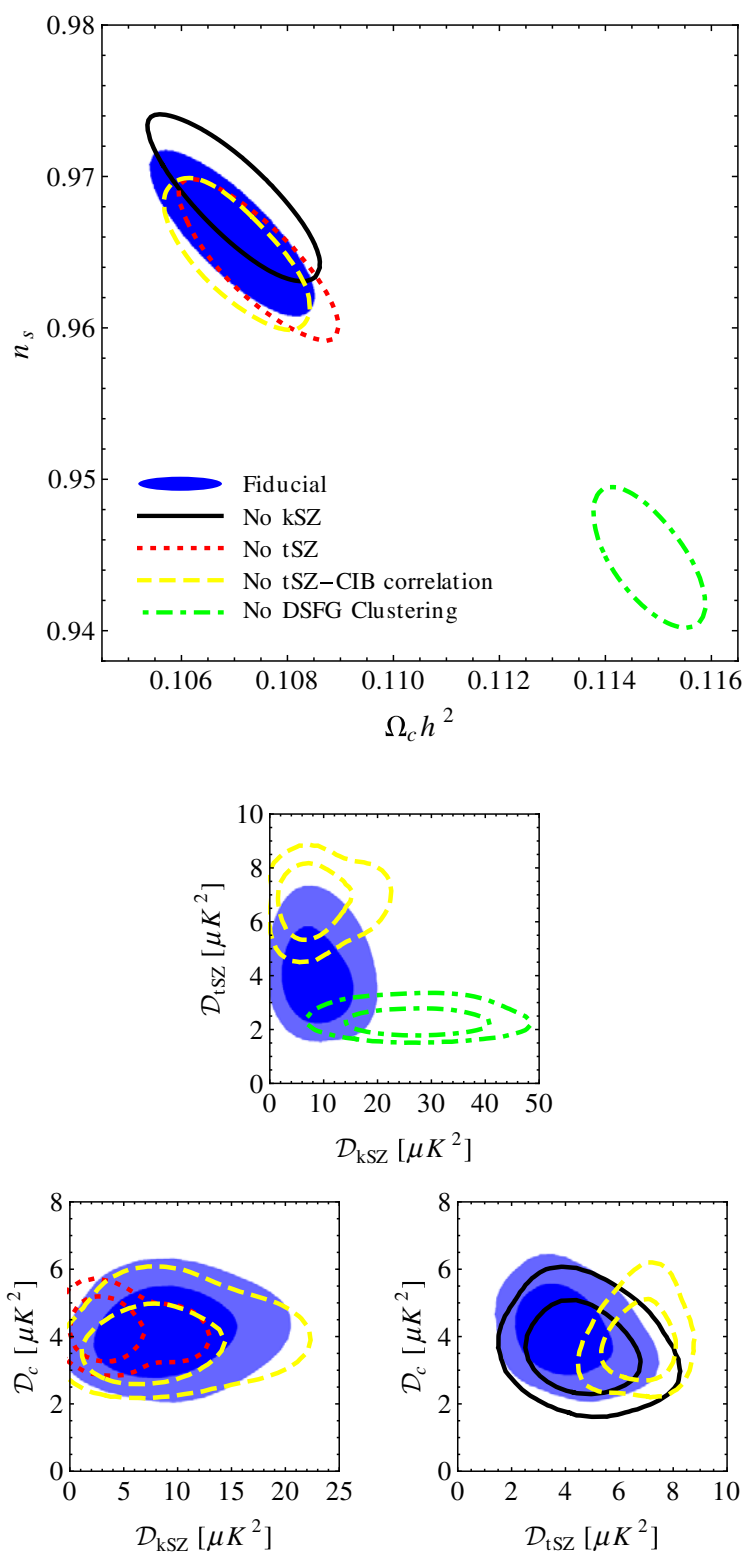

\begin{tabular}{l|c|c|c|c}
\hline \hline & Data & Analysis & $\Delta \chi^{2}$ & $N_{\ell}$ \\
\hline No tSZ & $\mathcal{D}_{t S Z}=4.3$ & $\mathcal{D}_{t S Z}=0$ & 43 & 6 \\
No kSZ & $\mathcal{D}_{k S Z}=4.2$ & $\mathcal{D}_{k S Z}=0$ & 2 & 1 \\
No DSFG Clustering & $\mathcal{D}_{C}=3.9$ & $\mathcal{D}_{C}=0$ & 791 & 1930 \\
No tSZ-DSFG Corr. & $r_{t S Z, C}=-.3$ & $r_{t S Z, C}=0$ & 3 & 1
\end{tabular}

Figure 6. Sixty-eight percent (and 95\% in the bottom panel) confidence contours for a suite of test cases examining the effect of neglecting to model different foregrounds. Unless explicitly stated above, other parameters were included in the data at their fiducial values listed in Table 2 and were marginalized over in the analysis. $N_{\ell}$ corresponds to the maximum number of $\ell$-bins per power spectrum one could use and still detect the error in modeling at $3 \sigma$ (see Section 7.1 for further discussion).

(A color version of this figure is available in the online journal.)

chains in this section include only Planck power spectra in the simulated data.

We expect the DSFG clustering to be extremely important to model since it is the second largest foreground contribution to the $\mathrm{CMB}$ linear combination in the $\ell$-range where Planck is most sensitive. When marginalized over, this contribution is constrained to be $10.5 \pm 0.6 \mu \mathrm{K}^{2}$ at $\ell=1500$, so setting it to zero is about an $18 \sigma$ change. The dot-dashed green contours in Figure 6 show that this is compensated by a systematic bias of $7 \sigma$ is $n_{s}$ and $11 \sigma$ in $\Omega_{c} h^{2}$, along with an increased $\mathrm{kSZ}$ power to about $30 \mu \mathrm{K}^{2}$. Using the middle panel of Figure 5 as a visual guide, we can examine how this happens. Though the $\mathrm{kSZ}$ increases to compensate for the missing clustering power at high- $\ell$, its shape is flatter than the DSFG contribution to the 


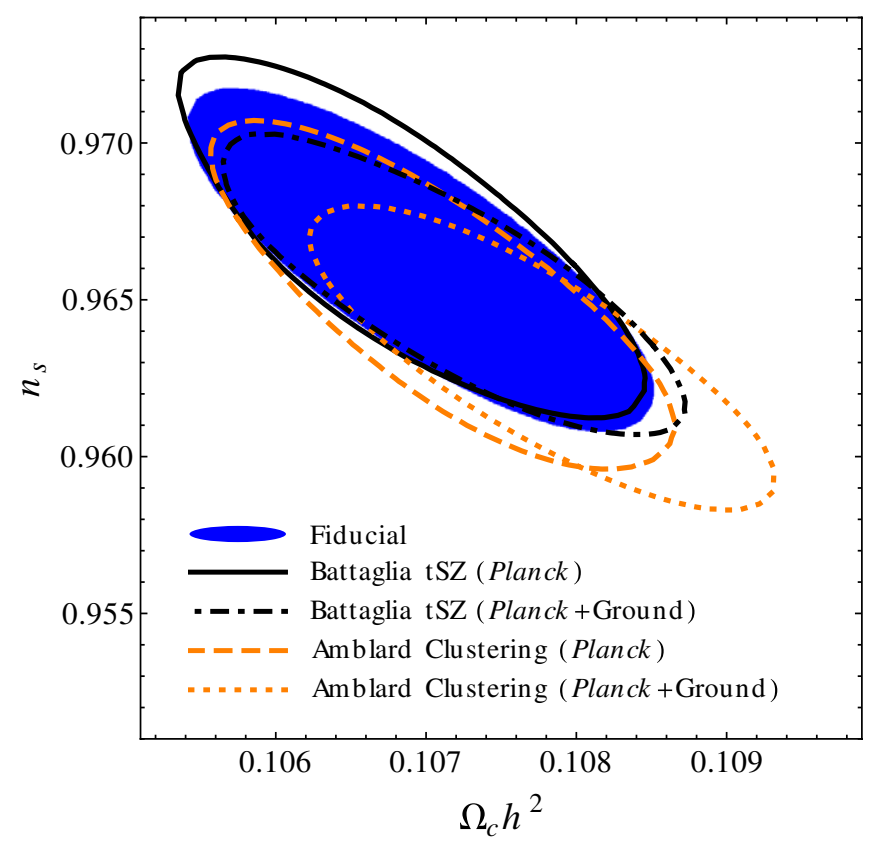

Figure 7. Effect on cosmological parameters from trying to fit our model to simulated data which includes (orange) the Battaglia et al. (2010) tSZ template and (black) the Amblard \& Cooray (2007) clustering template. These two models are the most dissimilar to ours, and thus show that our model can protect against biases of a few percent up to Planck sensitivity. The inclusion of Ground data necessitates more detailed modeling of only the clustering.

(A color version of this figure is available in the online journal.)

CMB linear combination, so $n_{s}$ decreases to roughly remove the extra power at a low- $\ell$.

At $217 \mathrm{GHz}$, the kSZ power in our fiducial model is a factor of 20 times smaller than the DSFG clustering and is therefore (in terms of cosmological parameter estimation) less troublesome. However, due to its identical frequency dependence to the CMB, we do expect the amplitude of the kSZ signal to be degenerate with cosmological parameters. The solid black contours in Figure 6 demonstrate the bias introduced if the kSZ component is omitted from the foreground modeling. We find a roughly $0.5 \sigma$ bias in $n_{s}$ as it increases to try to fill in the missing $4.2 \mu \mathrm{K}^{2}$ of kSZ power. The DSFG and tSZ plane (bottom right panel of Figure 6) shows that the other foregrounds are largely unaffected.

The thermal SZ component is neither frequency independent nor does it contribute as much power as the DSFG clustering, so we do not expect a bias as large as in either previous case. It does, however, project into the CMB linear combination to an $\ell$-shape very similar to the CMB itself (see the middle panel of Figure 5), making it more likely to be degenerate with cosmological parameters. From the results, we see about $0.3 \sigma$ biases in each of $n_{s}, \Omega_{c} h^{2}$, and $\Omega_{b} h^{2}$.

Finally, we consider neglecting a $30 \%$ tSZ-DSFG correlation, a value on the high end of expected correlation, but still consistent with Shirokoff et al. (2010). We expect this to have the smallest effect on the cosmological parameters since the power contribution is sub-dominant to all of the other foreground components at all frequencies which appear in the CMB linear combination at $>1 \%$. While the measured tSZ amplitude is biased at a few sigma as it raises to compensate for the missing power, the effect is not large enough to significantly impact any of the cosmological parameters.

One question is whether any of these analysis errors would be caught by a goodness-of-fit test. To address this question we present $\Delta \chi^{2}$ values in the table in Figure 7 . We can expect rms fluctuations in $\chi^{2}$ to be $\sqrt{N_{b}}$ where $N_{b}$ is the total number of bandpowers which is roughly equal to the number of degrees of freedom. If one is searching for signs of a contaminant that is very slowly varying in $\ell$, then one would bin coarsely to reduce the statistical fluctuations in $\chi^{2}$, to make a more stringent goodness-of-fit test.

We define $N_{\ell}$ to be the number of $\ell$-space bins such that the absolute $\Delta \chi^{2}$ from the fit is $99.7 \%$ inconsistent with random fluctuations. Thus we have $N_{\ell}=\left(\Delta \chi^{2}\right)^{2} /\left(9 \times N_{\text {spec }}\right)$ where $N_{\text {spec }}$ is the number of power spectra (36 here). We see that binning would not have to be coarse at all to detect the poor fit caused by neglecting clustering. We also see that for the other entries in the table, binning would have to be extremely coarse for the fits to be noticeably poor. Indeed, the binning would have to be coarser than is practical since the signals of interest, as well as the contaminants, would vary significantly across a bin. We conclude that only the "no clustering" case would produce a noticeably bad fit for Planck only.

\subsection{Modeling Sufficiency}

Given the demonstrated importance of the foreground components, we would now like to see if our modeling is sufficient to protect the cosmological parameters from biases if we have modeled the components, but modeled them incorrectly. In this section we consider the DSFG clustering and the tSZ effect.

For the DSFG clustering, we turn to the models plotted in Figure 1. Our parameterization should have the most trouble reproducing the Amblard \& Cooray (2007) model, which switches to a power law (as a consequence of nonlinear clustering) at $\ell \approx 2500$ rather than at $\ell=1500$ as in our fiducial model. The orange contours in Figure 7 show the results obtained when fitting our model to simulated power spectra that assume the Amblard \& Cooray (2007) clustering template. As we had hoped, for the case of Planck only (solid lines), there is no significant biasing.

We also explore the ability of our tSZ principal component model (based on the analytic model of Shaw et al. 2010) to encompass the variations in the tSZ models shown in Figure 2. We elect the Battaglia et al. (2010) one as the most dissimilar, since it lacks the effects of radiative cooling, and should be the most difficult for the Shaw et al. (2010) model to reproduce. Despite these differences, Figure 7 shows that for Planck the model is sufficient to encompass the shape uncertainty and protect cosmological parameters.

When we add in Ground (dashed lines), the requirements on the modeling accuracy are more stringent. For the clustering case, we see an almost $1 \sigma$ bias from using our fiducial model when the true model is the Amblard \& Cooray (2007) clustering template. Analyses with current data can tolerate much more discrepant clustering shapes (Dunkley et al. 2011). For future Planck+Ground analyses, the clustering shape will need to be modeled more accurately. For tSZ the modeling appears to be more robust; tSZ-induced biases are small even in the Planck+Ground case.

\subsection{Statistical Error Increase with and without Auxiliary Data}

We have demonstrated the possibility of $\sigma$-level biases in cosmological parameters arising from failure to model foregrounds. To prevent these biases, the foregrounds need to be jointly estimated or marginalized over. We now turn to two questions: (1) How much do the cosmological parameter statistical errors degrade due to foreground uncertainty? And (2) how much can 
Table 4

Statistical Error Degradation

\begin{tabular}{lcccccccccc}
\hline \hline & $10^{4} \Omega_{b} h^{2}$ & $10^{3} \Omega_{c} h^{2}$ & $10^{4} \Theta$ & $10^{3} n_{s}$ & $\ln \left(10^{10} A_{s}\right)$ & $\mathcal{D}_{D}$ & $\mathcal{D}_{R}$ & $\mathcal{D}_{C}$ & $\mathcal{D}_{\mathrm{tSZ}}$ & $\mathcal{D}_{\mathrm{kSZ}}$ \\
\hline Planck (fgs fixed) & 1.1 & 1.0 & 2.6 & 3.0 & 1.3 & $\ldots$ & $\ldots$ & $\ldots$ & $\ldots$ & $\ldots$ \\
Planck (fgs marginalized) & 1.2 & 1.0 & 2.6 & 3.6 & 1.4 & 3.4 & 6.0 & 1.3 & 1.0 & 4.4 \\
Planck+Ground & 1.1 & 1.0 & 2.6 & 3.3 & 1.3 & 0.3 & 3.0 & 0.5 & 0.6 & 2.6 \\
Planck (Clean DSFG) & 1.1 & 1.0 & 2.6 & 3.3 & 1.3 & 2.0 & 6.0 & & 1.0 & 4.4 \\
\hline
\end{tabular}

Notes. Entries are $1 \sigma$ constraints. Dashes indicate the parameter was fixed, while blanks mean the parameter is not applicable to that case. The normalization parameters $\mathcal{D}_{x}$ are in units of $\mu \mathrm{K}^{2}$. The different cases correspond to the following. (fgs fixed) Fixing all of the foregrounds at their fiducial values. (fgs marginalized) Marginalization over our full foreground model. (+Ground) Also including Ground auto and cross spectra in the simulated data. (Clean DSFG) Assuming 90\% reduced clustering power due to cleaning from higher frequencies.

be gained from using other data to constrain foregrounds and thereby reduce that degradation?

The top two rows of Table 4 show the effect of marginalizing over our entire foreground model as opposed to fixing it at fiducial values. In each row, the difference from $100 \%$ is the percent of degradation due to foreground marginalization. The second row shows the degradation is limited to $20 \%$ for $n_{s}$ and $10 \%$ for $A_{s}$ and $\Omega_{b} h^{2}$. We see no degradation in $\tau$ and $r$ since they are mainly constrained by large scales where the extragalactic foregrounds we consider are negligible. The dark energy equation of state $w$ is unaffected because it is mainly constrained by our \pm 0.3 prior.

Ground data can help reduce this degradation by better constraining the foregrounds using auto and cross spectra that are more sensitive at small scales. The improvement from adding these to the simulated data is shown in the row labeled Planck+Ground. The measurement of DSFG shot noise is improved tenfold, with the clustering and SZ effects also tightened by a factor of two. The radio amplitude is improved through constraints on the spectral dependence, and could be further improved though a prior on $\gamma_{R}$ from Ground source counts. The effect on the cosmological parameters is to remove essentially all of the degradation we incurred from marginalizing over the foreground model.

Above about $300 \mathrm{GHz}$, the DSFGs are the dominant source of anisotropy power on all scales. Correlations with maps at these higher frequencies, for example, maps from Planck bands above $217 \mathrm{GHz}$ or Herschel, can be used to place tight constraints on the DSFG components, at the price of requiring more sophisticated modeling for the spectral dependence and shape. Even with such modeling, the correlations are no longer fully coherent across frequencies so there is a limit to how much of the DSFG power can be "cleaned out" of the lower frequency maps. Following results in Knox et al. (2001), who assume a redshift dependent gray-body emissivity density tracing the linear matter power spectrum, we assume that we could clean out $90 \%$ of DSFG clustering power at the lower frequencies. As in the previous case of adding in Ground data, this again is enough to eliminate nearly all of the degradation on cosmological parameters.

\section{CONCLUSIONS}

To make full use of Planck's very small statistical error on CMB power spectra out to $\ell \sim 2500$, without introducing significant bias in the cosmological parameters, we must include contributions from extragalactic foregrounds and secondaries in our model of the data. Here we have presented a model of these contaminants, based on the latest data and modeling developments, and demonstrated its ability to remove biases in an eight-parameter cosmological model. The foreground model has 17 parameters-many more than any extragalactic foreground model used in analysis of CMB data to date. Despite the large number of nuisance parameters, marginalizing over all of them only increases statistical uncertainties in the cosmological parameters by, at most $10 \%-20 \%$. Almost all of this degradation can be avoided by inclusion of ground-based data or higher-frequency Planck bands.

Our model includes Poisson components from both radio galaxies and DSFGs, a clustering component due to DSFGs, contributions to kSZ power from patchy reionization, as well as after reionization is complete, and $\mathrm{tSZ}$ power. If $\mathrm{kSZ}$ power and tSZ power are at our fiducial values (slightly higher than the preferred values given current high-resolution groundbased data) then ignoring them in an analysis of Planck data would produce small, almost negligible biases, to cosmological parameter estimates. On the other hand, ignoring the clustering of DSFGs, would lead to a very large bias in cosmological parameters.

To avoid having to marginalize over these 17 parameters every time a new cosmological model is analyzed, we broke our procedure up into a two-step process, with the first step independent of the model of the primary CMB power spectra. The second step is an analysis of the CMB power spectra estimated in the first step, with a small number of foreground template amplitude parameters to marginalize over. The shapes of these templates, and priors on their amplitudes, are also outputs of the first step. Only the second step needs to be repeated in order to get constraints on the parameters of a new model of the primary CMB power spectra.

Looking toward the near future, the model will definitely evolve, increasing the faithfulness with which it represents reality, as we gain more information from the CMB-dominated channels in Planck, higher-frequency Planck channels, higherresolution ground-based data (SPT, SPTpol, and ACTpol) and higher-resolution, higher-frequency space-based data (Herschel). One could easily use our foreground model to study potential biases in extensions of the primary cosmological model, to include, for example, departure of the helium mass fraction from the prediction of Big Bang Nucleosynthesis, or a difference in the number of effective neutrino species from the standard model value.

We benefited from conversations with A. Challinor, J. Dunkley, G. Efstathiout, F. Finelli, S. Gratton, W. Holzapfel, C. Reichardt, D. Scott, G. Ziemann, and G. de Zotti. Part of the research described in this paper was carried out at the Jet Propulsion Laboratory, California Institute of Technology, under 
a contract with the National Aeronautics and Space Administration. L.S. acknowledges the support of Yale University and NSF grant AST-1009811. L.K. and M.M. acknowledge support from NSF grant 0709498.

\section{APPENDIX A \\ PRINCIPAL COMPONENT ANALYSIS FOR POWER SPECTRA}

In this paper we use a PCA to reduce the dimensionality of the tSZ astrophysical parameter space (Section 2.2) and of the entire foreground contribution to the CMB linear combination (Section 6.2). Here we present in more detail the procedure used in those sections.

Given $n_{\text {sim }}$ realizations of an $n_{\ell}$-length power spectrum, drawn from a statistically significant sample of parameter space, we first form the $\left[n_{\ell} \times n_{\text {sim }}\right]$ matrix $Y$. In each column of $Y$ we place the deviation from the mean power spectrum for that realization. This matrix is then subject to a singular value decomposition,

$$
Y=U S V^{T},
$$

where the columns of $U$ contain the orthogonal basis vectors, $S$ is a diagonal matrix of the singular values, and the columns of $V$ are the principle component weights. The $i$ th realization can be written as

$$
y_{\ell}^{(i)}=\sum_{\mu} \Phi_{\ell}^{\mu} w_{\mu}^{(i)},
$$

where the singular value-weighted orthogonal basis vectors are

$$
\Phi_{\ell}^{\mu}=\frac{1}{\sqrt{n_{\text {sim }}}} U_{\ell \mu} S_{\mu \mu}
$$

and the $w_{\mu}$ are the weights:

$$
w_{\mu}^{(i)}=\sqrt{n_{\operatorname{sim}}} V_{i \mu} .
$$

Because the singular values are in decreasing order, we can truncate the sum in Equation (A2) at some small value of $\mu$ and still accurately describe each realization. Furthermore, the distribution of weights $P\left(w_{\mu}\right)$ sampled over $n_{\text {sim }}$ realizations provides a prior on our principal component amplitudes equivalent to the parameter space which was sampled to produce the $Y$ matrix.

\section{APPENDIX B}

\section{CMB LINEAR COMBINATION GENERALIZATION TO OFF-DIAGONAL CORRELATIONS}

The method for constructing a best estimate of the CMB presented in Section 6 assumes only temperature power spectra, and a covariance which is diagonal in $\ell$. The generalization to include polarization and mode-mode coupling induced by sky masking is presented here. The math is, in fact, identical for the two scenarios, so in this appendix we will refer to polarization types with the understanding that we could just as well be talking about different values of $\ell$.

The added difficulty in dealing with different power spectrum types (for simplicity here just TT and EE) comes from the fact that we cannot arbitrarily create linear combinations which sum them. For example, $C^{\prime}=C_{100 \mathrm{GHz}}^{\mathrm{TT}}-C_{100 \mathrm{GHz}}^{\mathrm{EE}}$ neither preserves $\mathrm{CMB}$ normalization nor can we be sure it is CMB-free independent of model. To remedy this, we make sure that in our construction, any linear combination we consider must have the $\mathrm{CMB}$ signal cancel out for all but one type. For example, $C^{\prime}=C_{100 \mathrm{GHz}}^{\mathrm{TT}}-C_{100 \mathrm{GHz}}^{\mathrm{EE}}+C_{143 \mathrm{GHz}}^{\mathrm{EE}}$ is a valid linear combination.

We start by considering the covariance matrix for the TT and EE spectra:

$$
\left[\begin{array}{cc}
\Sigma_{\mathrm{TT}} & \cdots \\
\cdots & \Sigma_{\mathrm{EE}}
\end{array}\right]
$$

By creating the single-type weight matrix (Equation (29)) for each of the diagonal blocks, we can cancel the CMB out of all but two weightings. The new covariance will look like

$$
\begin{gathered}
{\left[\begin{array}{cc}
W_{\mathrm{TT}}^{T} & 0 \\
0 & W_{\mathrm{EE}}^{T}
\end{array}\right]\left[\begin{array}{cc}
\Sigma_{\mathrm{TT}} & \cdots \\
\cdots & \Sigma_{\mathrm{EE}}
\end{array}\right]\left[\begin{array}{cc}
W_{\mathrm{TT}} & 0 \\
0 & W_{\mathrm{EE}}
\end{array}\right]} \\
=\left[\begin{array}{cc}
\left(\begin{array}{cc}
\sigma_{\mathrm{TT}} & 0 \\
0 & \ddots
\end{array}\right) & \cdots \\
\cdots & \left(\begin{array}{cc}
\sigma_{\mathrm{EE}} & 0 \\
0 & \ddots
\end{array}\right)
\end{array}\right]
\end{gathered}
$$

Under a permutation to place the two CMB weightings at the front, the covariance becomes

$$
\left[\begin{array}{cc}
\left(\begin{array}{cc}
\sigma_{\mathrm{TT}} & \cdots \\
\cdots & \sigma_{\mathrm{EE}}
\end{array}\right) & \cdots \\
\cdots & \ddots
\end{array}\right] \equiv\left[\begin{array}{cc}
\Sigma_{\mathrm{cmb}} & \Sigma_{\mathrm{cross}}^{T} \\
\Sigma_{\mathrm{cross}} & \Sigma_{\mathrm{diff}}
\end{array}\right],
$$

where we have labeled $\Sigma_{\mathrm{cmb}}$ as the covariance between TT and EE estimates, $\Sigma_{\text {diff }}$ as the covariance of the CMB-free differenced spectra, and $\Sigma_{\text {cross }}$ as the cross-correlation between the two. We now would like to do one final reweighting in an attempt to zero out the cross-correlation. The reweighting should leave the differenced spectra unchanged, should not add TT and $\mathrm{EE}$ together, but will add $\mathrm{CMB}$ and $\mathrm{CMB}$-free power spectra. Note that this will continue to satisfy our earlier condition that all but one $\mathrm{CMB}$ type canceling out. The reweighting matrix will look like

$$
\left[\begin{array}{cc}
I & 0 \\
W^{\prime} & I
\end{array}\right]
$$

The new covariance must satisfy

$$
\left[\begin{array}{cc}
I & W^{\prime T} \\
0 & I
\end{array}\right]\left[\begin{array}{cc}
\Sigma_{\mathrm{cmb}} & \Sigma_{\mathrm{cross}}^{T} \\
\Sigma_{\mathrm{cross}} & \Sigma_{\mathrm{diff}}
\end{array}\right]\left[\begin{array}{cc}
I & 0 \\
W^{\prime} & I
\end{array}\right]=\left[\begin{array}{cc}
\Sigma_{\mathrm{cmb}}^{\prime} & 0 \\
0 & \Sigma_{\text {diff }}
\end{array}\right]
$$

Solving for $W^{\prime}$ yields

$$
W^{\prime}=-\Sigma_{\text {diff }}^{-1} \Sigma_{\text {cross }}
$$

\section{REFERENCES}

Amblard, A., \& Cooray, A. 2007, ApJ, 670, 903

Amblard, A., Cooray, A., Serra, P., et al. 2011, Nature, 470, 510

Amblard, A., \& White, M. 2005, New Astron., 10, 417

Battaglia, N., Bond, J. R., Pfrommer, C., Sievers, J. L., \& Sijacki, D. 2010, ApJ, 725,91

Battye, R. A., Browne, I. W. A., Peel, M. W., Jackson, N. J., \& Dickinson, C. 2011, MNRAS, 413, 132

Bond, J. R., Carr, B. J., \& Hogan, C. J. 1986, ApJ, 306, 428

Bond, J. R., Carr, B. J., \& Hogan, C. J. 1991, ApJ, 367, 420

Bouchet, F. R., \& Gispert, R. 1999, New Astron., 4, 443

Cardoso, J., Martin, M., Delabrouille, J., Betoule, M., \& Patanchon, G. 2008 arXiv:0803.1814

Carlstrom, J. E., Holder, G. P., \& Reese, E. D. 2002, ARA\&A, 40, 643 
Challinor, A., \& Lewis, A. 2005, Phys. Rev. D, 71, 103010

de Zotti, G., Ricci, R., Mesa, D., et al. 2005, A\&A, 431, 893

Duffy, A. R., Battye, R. A., Davies, R. D., Moss, A., \& Wilkinson, P. N. 2008, MNRAS, 383, 150

Dunkley, J., Hlozek, R., Sievers, J., et al. 2011, ApJ, 739, 52

Fendt, W. A., \& Wandelt, B. D. 2007, arXiv:0712.0194

Fixsen, D. J., Dwek, E., Mather, J. C., Bennett, C. L., \& Shafer, R. A. 1998, ApJ, 508,123

Giavalisco, M., Steidel, C. C., Adelberger, K. L., et al. 1998, ApJ, 503, 543

Giodini, S., Pierini, D., Finoguenov, A., et al. 2009, ApJ, 703, 982

Gold, B., Odegard, N., Weiland, J. L., et al. 2011, ApJS, 192, 15

Gruzinov, A., \& Hu, W. 1998, ApJ, 508, 435

Haiman, Z., \& Knox, L. 2000, ApJ, 530, 124

Hall, N. R., Keisler, R., Knox, L., et al. 2010, ApJ, 718, 632

Hodge, J. A., Becker, R. H., White, R. L., Richards, G. T., \& Zeimann, G. R. 2011, AJ, 142, 3

Hu, W. 2000, ApJ, 529, 12

Hughes, D. H., Serjeant, S., Dunlop, J., et al. 1998, Nature, 394, 241

Iliev, I. T., Pen, U., Bond, J. R., Mellema, G., \& Shapiro, P. R. 2007, ApJ, 660, 933

Keisler, R., Reichardt, C. L., Aird, K. A., et al. 2011, ApJ, 743, 28

Knox, L. 1999, MNRAS, 307, 977

Knox, L., Cooray, A., Eisenstein, D., \& Haiman, Z. 2001, ApJ, 550, 7

Knox, L., Holder, G. P., \& Church, S. E. 2004, ApJ, 612, 96

Knox, L., Scoccimarro, R., \& Dodelson, S. 1998, Phys. Rev. Lett., 81, 2004

Komatsu, E., \& Kitayama, T. 1999, ApJ, 526, L1

Komatsu, E., \& Seljak, U. 2002, MNRAS, 336, 1256

Komatsu, E., Smith, K. M., Dunkley, J., et al. 2010, arXiv:1001.4538

Lagache, G., Bavouzet, N., Fernandez-Conde, N., et al. 2007, ApJ, 665, L89

Lau, E. T., Kravtsov, A. V., \& Nagai, D. 2009, ApJ, 705, 1129

Leach, S. M., et al. 2008, A\&A, 491, 597

Lewis, A., \& Bridle, S. 2002, Phys. Rev. D, 66, 103511

Lewis, A., Challinor, A., \& Lasenby, A. 2000, ApJ, 538, 473

Lueker, M., Reichardt, C. L., Schaffer, K. K., et al. 2010, ApJ, 719, 1045

McQuinn, M., Furlanetto, S. R., Hernquist, L., Zahn, O., \& Zaldarriaga, M. 2005, ApJ, 630, 643

Navarro, J. F., Frenk, C. S., \& White, S. D. M. 1997, ApJ, 490, 493

Ostriker, J. P., \& Vishniac, E. T. 1986, ApJ, 306, L51
Peacock, J. A., \& Dodds, S. J. 1996, MNRAS, 280, L19

Peel, M. W., Battye, R. A., \& Kay, S. T. 2009, MNRAS, 397, 2189

Planck Collaboration. 2006, arXiv:astro-ph/0604069

Planck Collaboration. 2011a, A\&A, 536, A8

Planck Collaboration. 2011b, A\&A, 536, A9

Planck Collaboration. 2011c, A\&A, 536, A10

Planck Collaboration. 2011d, A\&A, 536, A13

Planck Collaboration. 2011e, A\&A, 536, A18

Prunet, S., Sethi, S. K., Bouchet, F. R., \& Miville-Deschenes, M. 1998, A\&A, 339, 187

Puget, J., Abergel, A., Bernard, J., et al. 1996, A\&A, 308, L5

Righi, M., Hernández-Monteagudo, C., \& Sunyaev, R. A. 2008, A\&A, 478, 685

Santos, M. G., Cooray, A., Haiman, Z., Knox, L., \& Ma, C. 2003, ApJ, 598, 756

Sazonov, S. Y., \& Sunyaev, R. A. 1999, MNRAS, 310, 765

Scott, D., \& White, M. 1999, A\&A, 346, 1

Sehgal, N., Bode, P., Das, S., et al. 2010, ApJ, 709, 920

Serra, P., Cooray, A., Amblard, A., Pagano, L., \& Melchiorri, A. 2008, Phys. Rev. D, 78, 043004

Shaw, L. D., Nagai, D., Bhattacharya, S., \& Lau, E. T. 2010, ApJ, 725, 1452

Shaw, L. D., Zahn, O., Holder, G. P., \& Doré, O. 2009, ApJ, 702, 368

Shirokoff, E., Reichardt, C. L., Shaw, L., et al. 2010, arXiv:1012.4788

Smith, R. E., Peacock, J. A., Jenkins, A., et al. 2003, MNRAS, 341, 1311

Taburet, N., Aghanim, N., Douspis, M., \& Langer, M. 2009, MNRAS, 392, 1153

Tegmark, M., \& Efstathiou, G. 1996, MNRAS, 281, 1297

Tegmark, M., Eisenstein, D. J., Hu, W., \& de Oliveira-Costa, A. 2000, ApJ, 530, 133

Tinker, J., Kravtsov, A. V., Klypin, A., et al. 2008, ApJ, 688, 709

Trac, H., Bode, P., \& Ostriker, J. P. 2010, arXiv:1006.2828

Vieira, J. D., Crawford, T. M., Switzer, E. R., et al. 2010, ApJ, 719, 763

Viero, M. P., Ade, P. A. R., Bock, J. J., et al. 2009, ApJ, 707, 1766

Vishniac, E. T. 1987, ApJ, 322, 597

White, M., Hernquist, L., \& Springel, V. 2002, ApJ, 579, 16

Wright, E. L., Chen, X., Odegard, N., et al. 2009, ApJS, 180, 283

Zahn, O., Mesinger, A., McQuinn, M., et al. 2010, arXiv:1003.3455

Zahn, O., Zaldarriaga, M., Hernquist, L., \& McQuinn, M. 2005, ApJ, 630, 657

Zhang, P., Pen, U., \& Trac, H. 2004, MNRAS, 347, 1224

Zhang, P., \& Sheth, R. K. 2007, ApJ, 671, 14 